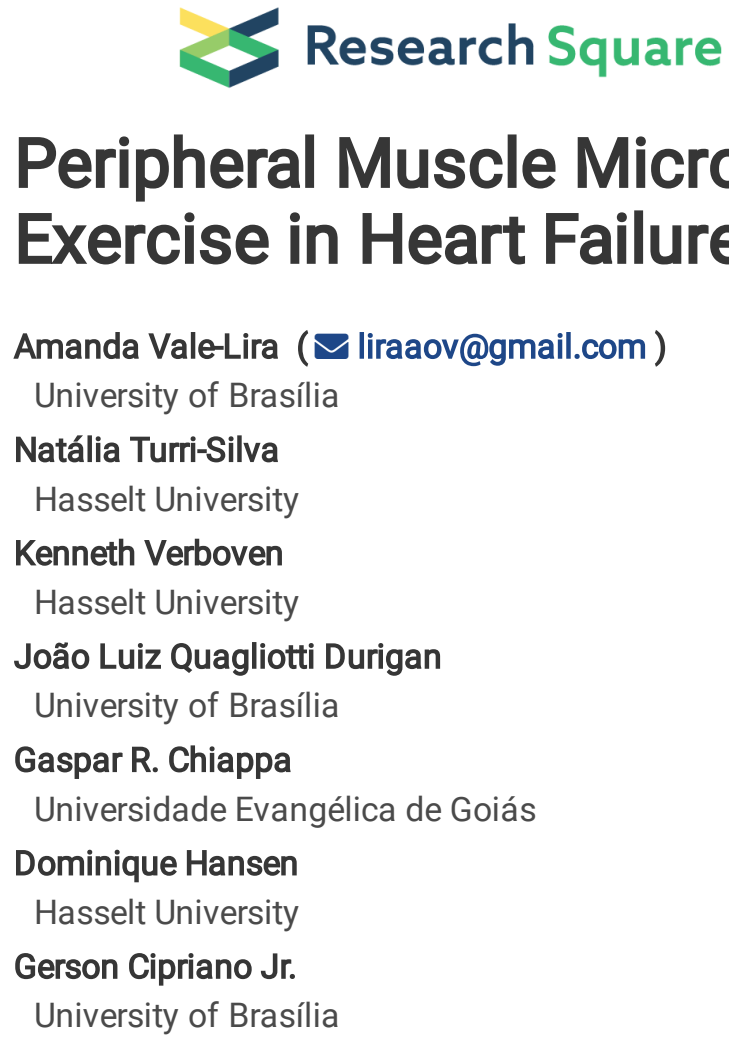

\title{
Peripheral Muscle Microcirculation Dynamics During Strength Exercise in Heart Failure Patients: A Cross-Sectional Study
}

Research Article

Keywords: near-infrared spectroscopy (NIRS), heart failure

Posted Date: July 8th, 2021

DOI: https://doi.org/10.21203/rs.3.rs-677335/v1

License: (c) (i) This work is licensed under a Creative Commons Attribution 4.0 International License. Read Full License 


\section{Abstract}

This cross-sectional study aimed to compare the muscle microcirculation dynamics via near-infrared spectroscopy (NIRS) response during an isokinetic muscle strength test and ultrasound-derived parameters in heart failure patients with preserved or reduced ejection fraction phenotypes (HFpEF and HFrEF, respectively) and different functional severities (Weber Class A, B, and C). Twenty-eight aged-matched patients with $\operatorname{HFpEF}(n=16)$ and $\operatorname{HFrEF}(n=12)$ were assessed. Within HFpEF, Weber Class $C$ patients presented a worse capability to reach a greater oxygen extraction during exercise than Classes $\mathrm{A}$ and $\mathrm{B}$. Among those with Weber $C$ severity, while HFrEF patients reached lower oxyhemoglobin $\left(\mathrm{O}_{2} \mathrm{Hb}, \mu \mathrm{M}\right)(-10.9 \pm 3.8$ vs. $-23.7 \pm 5.7, \mathrm{p}=0.029)$ during exercise, $\mathrm{HFpEF}$ kept a lower $\mathrm{O}_{2} \mathrm{Hb}$ during the recovery period ( $-3.0 \pm 3.4$ vs. $\left.5.9 \pm 2.8, \mathrm{p}=0.007\right)$. Among the ultrasound-derived variables, HFpEF, Weber Class C, also presented a higher echo intensity than HFrEF patients (29.7 \pm 8.4 vs. $15.1 \pm 6.8, p=0.017)$. Our study reveals more pronounced impairments in local microcirculatory dynamics in HFpEF vs. HFrEF patients during muscle strength exercise, combined with muscle-skeletal abnormalities detected via ultrasound imaging, which may help to explain the commonly observed exercise intolerance in HFpEF patients.

\section{Introduction}

Cardiovascular disease (CVD) is the most important cause of premature mortality, leading to over 17 million deaths yearly around the globe [1-3]. Heart failure (HF) ranks as the second most prevalent CVD [4], exhibiting typical clinical symptoms (i.e., breathlessness, fatigue, ankle swelling) or signs (i.e., pulmonary crackles, peripheral edema) in association with key structural and functional cardiac abnormalities [5]. Left ventricular ejection fraction (LVEF) assessment using transthoracic echography has been utilized to distinguish patients with preserved or reduced ejection fraction phenotype (HFpEF and HFrEF, respectively). This differentiation is required due to different etiologies, demographics, comorbidities, and therapeutic responses [5]. HFpEF and HFrEF patients typically present similar cardiorespiratory fitness reductions and worse morbidity, hospitalization, and five-year mortality rates compared to healthy individuals [6,7]. Although HFrEF has been widely explored, approximately one-half of all patients exhibit a preserved ejection fraction $\geq$ of $50 \%$, thus reinforcing the need for a better understanding of exercise intolerance in HFpEF [8].

Although peripheral mechanisms have been related to exercise tolerance in HF patients [9-11], its etiology remains poorly understood in HFpEF [7,12]. As the muscular component has become a key factor in these peripheral mechanisms, it may differ according to the HF phenotype $[5,13,14]$. The muscular microcirculatory contributions to exercise tolerance amongst different $\mathrm{HF}$ phenotypes are poorly understood, particularly when submitted to muscular stress. A better understanding of muscular microcirculatory dynamics is pivotal to unravel the integrative pathophysiology of exercise intolerance in these patients.

Skeletal muscle dysfunction is widespread in HF patients, in which sarcopenia, i.e., the loss of muscle strength and muscle mass, plays an important role $[15,16]$. Considering the skeletal muscle plasticity and the potential for peripheral adaptation when following exercise-based interventions, changes related to muscle function (such as mass, strength, quality, or microvascular function) may be more noticeable when compared to changes in cardiopulmonary variables $[17,18]$. Previous research demonstrated that peripheral skeletal muscle dysfunction majorly affects the exercise tolerance in HFrEF $[10,17,19-21]$, while in $\mathrm{HFpEF}$, muscle microvascular dysfunction and oxygenation seem to be more pronounced $[19,22]$ due to expected HF-related peripheral adaptations, such as vascular rarefactions, skeletal muscle abnormalities [23], and higher regional adipose distribution [24]. Of interest, skeletal muscle perfusion reduction in HFpEF may worsen the oxidative capacity and inflammatory stress, which have been strongly associated with disease-related muscle loss $[25,26]$. In this sense, assessing the muscle quality and local microcirculatory response differences among HFpEF and HFrEF patients and the association with muscle strength and cardiorespiratory fitness is clinically relevant [27], paving new secondary prevention and rehabilitation treatment alternatives [28].

This study primarily aims to explore the mechanisms leading to exercise intolerance by comparing the local oxygen extraction response during muscle strength exercise and ultrasound-derived parameters among different HF phenotypes with distinct disease severities. Secondarily, to analyze possible associations between exercise-induced microcirculation responses and ultrasoundderived variables with a) isokinetic muscle strength test, $b$ ) cardiorespiratory fitness, and c) peak power output. The outcome measures will be explored according to HF phenotypes and disease severities. 
We hypothesized that peripheral dysfunctions are more pronounced in patients with HFpEF compared to HFrEF because of a lower oxygen extraction capacity and poor muscle quality $[19,22]$.

\section{Methods}

\section{Study design and participants}

This is a cross-sectional observational study in which the assessors were not blinded for the different subgroups and following the STROBE recommendations [29]. The study was performed in accordance with the Declaration of Helsinki (2013) (approved by the ethical committee of the University of Brasília, CAAE 81309417.7.0000.8093). After a careful explanation of the nature and risks of the experimental procedures, all participating patients provided informed consent before starting the measurements. The study was realized between June 2018 and September 2019 at the University of Brasília.

Male and female individuals from a convenient sample, diagnosed with HFpEF or HFrEF, stable and under optimal medical treatment, were recruited and allocated by phenotype. The inclusion criteria were: 1) minimal age of 35 years; 2) at least six months of HFrEF or HFpEF diagnosis [5]; 3) HF with ischemic, hypertensive, or idiopathic etiology; 4) clinically stable for at least three months; and 5) a sedentary lifestyle (in the last six months). The exclusion criteria were: 1) clinically diagnosed pulmonary, inflammatory, musculoskeletal, or orthopedic diseases precluding exercise performance; and 2) functional New York Heart Association (NYHA) [30] class IV.

All participants were assessed during four experimental visits. The first visit was directed to clinical assessment, body composition, and pulmonary function; the second to echocardiogram assessment; and the third for muscle ultrasound and cardiopulmonary exercise testing. Finally, a fourth visit was planned to assess the isokinetic muscle strength and local oxygen with near-infrared spectroscopy (NIRS).

\section{Baseline clinical characteristics}

Patients were evaluated by a cardiologist who collected detailed information about the clinical history, diagnosis, and current symptoms. The NYHA [30] and Weber [31] functional classification were included to provide complementary clinical information regarding HF severity. The whole-body composition was assessed using dual-energy X-ray absorptiometry (DXA; Supplementary Material), cardiac function using echocardiography (Supplementary Material), pulmonary function via spirometry (Supplementary Material), and cardiorespiratory fitness via cardiopulmonary exercise test (CPX; Supplementary Material).

\section{Isokinetic muscle strength test}

Isokinetic muscle strength tests were performed using the Biodex system III Isokinetic Dynamometer (Biodex Medical, Inc., Shirley, NY). The axis of rotation of the dynamometer arm was adjusted to the right knee, and velcro belts were used to secure the thigh, pelvis, and trunk to the chair to prevent compensatory body movement. The lateral femoral epicondyle was used as the bony landmark for matching the knee joint with the axis of rotation of the dynamometer resistance adapter. Gravity correction was obtained by measuring the torque exerted on the dynamometer resistance adapter with the knee in a relaxed state at full extension. Patients were instructed to fully extend and flex the knee and work maximally during each exercise set. Verbal encouragement was given throughout the test session.

Isokinetic muscle strength assessment protocol comprised 20 repetitions, requiring maximum concentric effort required at an angular velocity of $180 \%$ s. Patients performed six initial submaximal repetitions for familiarization purposes. After three minutes of rest, the isokinetic muscle strength test was performed [32-37]. Variables analyzed were peak torque (Nm) and adjusted per body weight ratio $(\mathrm{Nm} . \mathrm{kg})$, total repetition maximum work $(\mathrm{J})$ and adjusted per body weight ratio (\%), total work (J), work fatigue (\%), and average power (W).

\section{Near-infrared spectroscopy (NIRS)}

During isokinetic muscle strength testing, a near-infrared spectroscopy (NIRS) device with a dual-wavelength (760 and $850 \mathrm{~nm}$ ), continuous-wave system type, containing three pairs of LEDs configured for spatially resolved spectroscopy (SRS) with a sourcedetector spacing of 30,35, and $40 \mathrm{~mm}$ were utilized to assess local oxygen extraction response (Portamon for OxySoft 3.0.95, 
Artinis Medical Systems, Amsterdam, Netherlands). Changes in absorbance were recorded using the oxyhemoglobin $\left(\mathrm{O}_{2} \mathrm{Hb}, \mu \mathrm{M}\right)$ and deoxyhemoglobin $(\mathrm{HHb}, \mu \mathrm{M})$ values to assess the oxygenation status of the muscle [38]. In addition, the tissue saturation index (TSI, \%) was calculated from the absorption of coefficients derived from the attenuation of light at different source-detector distances and wavelengths as a relative value (\%), being feasible for comparing and evaluating the achievement of critical limits during exercise. For this, the equipment was positioned on the right leg vastus lateralis (approximately $5 \mathrm{~cm}$ from the lateral patellar border), covered with a dark blue elastic band to avoid interference from ambient light and adhesive tape without pressing the equipment. The data were sampled at $10 \mathrm{~Hz}$ and stored for offline analysis using the LabChart Pro v8 software (ADInstruments, Sidney, Australia).

For statistical analysis and graph signal processing analysis of the NIRS curve, baseline (mean obtained value for the 30 s of the resting phase), exercise (lowest obtained value for $\mathrm{TSI}, \%$, and, $\mathrm{O}_{2} \mathrm{Hb}, \mu \mathrm{M}$ and highest for the $\mathrm{HHb}, \mu \mathrm{M}$ ) with a maximum interval variation acceptance of 4 seconds (20 to 24s, depending on manual NIRS mark) and recovery (highest obtained value for TSI, \% and $\mathrm{O}_{2} \mathrm{Hb}, \mu \mathrm{M}$ and lowest for the $\mathrm{HHb}, \mu \mathrm{M}$ ) were considered as time points for comparison [25;39]. An individual visual inspection of the curves was made to exclude possible failures/noise from the graph signal. Lately, eligible individuals were analyzed and presented on graphs that included the individual mean values from each variable (representative cases).

\section{Ultrasound-derived measures: echo intensity and muscle thickness}

The ultrasound images were captured by using an ultrasound device (HD11XE, Phillips, Amsterdam, Netherlands) with a 7.5MHz linear matrix transducer. The individuals were evaluated in a supine position with the knee in passive flexion with a $15 \mathrm{~cm}$ underknee support and neutral rotation. The images were always acquired on the right leg with the transducer placed transverse and perpendicular to the long axis of the anterior thigh, rectus femoris (RF), and vastus lateralis (VL) muscles ( $50 \%$ of the distance between the iliac spine anterior superior to the superior edge of the patella) to assess muscle thickness, using appropriate transmission gel [40]. The images were analyzed using the ImageJ software (1.52q version, Bethesda, EUA)[41]. The quadriceps femoris was analyzed between the uppermost part of the femur and the superficial fascia of the rectus femoris (which includes the rectus femoris and vastus intermedius) and the isolated rectus femoris $[42,43]$. The measurement of echo intensity was determined by a gray-scale analysis using ImageJ software. The region of interest was selected for each assessed muscle, including all muscle areas and removing bone or surrounding fascia from the selected area [42]. The mean was calculated using an 8-bit resolution measure, resulting in a number between $0=$ black and $255=$ white. An average of the three measurements per muscle was calculated. In the quadriceps femoris, only the rectus femoris muscle was used for analysis [42,43]. Patients were instructed not to perform any physical activities 24 hours before testing.

\section{Statistical analysis}

Data are expressed as mean \pm standard deviation (SD), absolute (n), or relative frequencies (\%). Shapiro-Wilk test was used to indicate sample data distribution. Parametric or non-parametric tests were applied accordingly. Group differences for continuous outcome variables were compared using unpaired $t$ (mean difference and 95\% confidence interval) or Mann-Whitney U test (Hodges-Lehmann's difference). Categoric variables were compared using Fisher's exact test.

We performed a bivariate correlation (Spearman's or Pearson's) analysis to investigate the associations between muscle microcirculation dynamics ( $\mathrm{TSI}, \% ; \mathrm{O}_{2} \mathrm{Hb}, \mu \mathrm{M}$ and $\mathrm{HHb}, \mu \mathrm{M}$ ) and echo intensity (El, 0-255) with isokinetic muscle strength (PT, Nm), cardiorespiratory fitness (peak $\mathrm{VO}_{2}, \mathrm{~mL} \mathrm{~kg}^{-1} \cdot \mathrm{min}^{-1}$ ) and peak power output (Watts) among HF phenotypes (HFpEF and HFrEF) and severity of functional impairment classification (Weber $A+B$ and Weber $C$ ).

Moreover, to verify possible association among muscular thickness $(\mathrm{cm})$ with muscle strength (peak torque, PT, Nm), cardiorespiratory fitness (peak $\mathrm{VO}_{2}, \mathrm{~mL} \mathrm{~kg}^{-1} \cdot \mathrm{min}^{-1}$ ), and peak power output (W). Association levels was defined according to correlation coefficient ( $r$ ) ( 0.00 no association; 0.20 weakly; 0.50 moderately; 0.8 strongly and 1.00 perfectly) [44] or (rho) ( 0.00 to 0.20 negligible; 021 to 0.40 weak; 0.41 to 0.60 moderate; 0.61 to 0.80 strong and 0.81 to 1.00 very strong) [45].

Considering the absence of similar studies involving microcirculatory dynamics withing resistance exercise in heart failure, the calculation of sample size was not possible. Because of this, we decided to run the post-hoc analysis in order to detect the power calculation of the study (effect size). By considering an alpha error of 0.05 , and the power (1-beta) 0.80 for the parameter $\mathrm{O}_{2} \mathrm{Hb}$ 
during the exercise period on Weber $\mathrm{C}$ in between phenotypes, we have an effect size result of 2.47 (high); for the parameter $\mathrm{O}_{2} \mathrm{Hb}$ during the recovery period on Weber $\mathrm{C}$ in between phenotypes, we have an effect size result of 2.38 (high); for the parameter $\mathrm{HHb}$ during the recovery period on Weber $A+B$ in between phenotypes, we have an effect size result of 2.31 (high); for the parameter $\mathrm{O}_{2} \mathrm{Hb}$ during the exercise period on Weber $\mathrm{A}+\mathrm{B}$ in HFpEF group, we have an effect size result of 2.07 (high); and for the parameter $\mathrm{HHb}$ during the recovery period on Weber $\mathrm{A}+\mathrm{B}$ in HFrEF group, we have an effect size result of 2.68 (high). These parameters was chosen because its statistical difference is $<0.05$. The effect size for group comparisons was estimated using G*Power Software 3.1 .

Statistical software GraphPad Prism (8.4.0, California, San Diego) was used for statistical analyses and figure production. All analyses considered $95 \%$ confidence interval $(\mathrm{Cl})$ and statistically significant was set at p-value $\leq 0.05$ (two-tailed).

\section{Results}

\section{Baseline Clinical characteristics}

Participants' characteristics are shown in Table 1 and supplementary material (Table S1). Both groups were similar by design in terms of age and BMI when comparing both phenotypes by Weber class $(p>0.05)$. There were no differences $(p>0.05)$ between risk factors and CVD's in both phenotypes (Table S1). However, HFpEF patients used fewer diuretics compared to HFrEF patients $(p<0.05)$. As expected, differences were detected for all echocardiographic parameters between HF phenotypes $(p<0.05)$ (Table 1). Regarding the cardiorespiratory fitness (Table 1), subjects presented similar peak $\mathrm{VO}_{2}\left(\mathrm{~mL} \cdot \mathrm{kg}^{-1} \cdot \mathrm{min}^{-1}\right)$ and VE/VCO 2 Slope $(p<0.05)$, independently of phenotype or disease severity. However, HFpEF presented a higher peak power output (W), predicted peak $\mathrm{VO}_{2}(\%)$, and peak $\mathrm{VO}_{2}\left(\mathrm{~mL} \cdot \mathrm{min}^{-1}\right)$ than the HFrEF group $(\mathrm{p}<0.05$, respectively). Considering Weber Class, HFpEF with Weber Class $\mathrm{A}+\mathrm{B}$ patients presented a higher absolute peak $\mathrm{VO}_{2}\left(\mathrm{~mL} \cdot \mathrm{min}^{-1}\right)$ and peak power output $(\mathrm{W})$ as opposed to $\operatorname{HFrEF}(p<0,05)$. Fat mass and lean tissue distribution were similar between subgroups $(p>0.05)$ (Table S1). Pulmonary function variables (Table S1) were not different $(p<0.05)$ when comparing both phenotypes (HFrEF and HFpEF) although comparing severity classification, only Weber Class $C$ presented a lower predictive value of $\mathrm{FEV}_{1}(p=0.24)$ and $\mathrm{FEV}_{1} / \mathrm{FVC}$ ratio $(p=0.020)$ on HFpEF than HFrEF group. 
Table 1

Demographic, anthropometric, and clinical characteristics in both heart failure phenotypes.

\begin{tabular}{|c|c|c|c|c|c|c|c|c|c|}
\hline \multirow{3}{*}{ Parameters } & \multicolumn{2}{|c|}{ HFpEF $(n=16)$} & \multicolumn{2}{|c|}{ HFrEF $(n=12)$} & \multicolumn{2}{|c|}{ HFpEF vs. HFrEF } & \multicolumn{2}{|c|}{$\begin{array}{l}\text { Weber Class A+ B } \\
\text { vs. } \\
\text { Weber Class C }\end{array}$} & \multirow{3}{*}{$\begin{array}{l}\text { HFpEF } \\
\text { vs. } \\
\text { HFrEF } \\
\text { p-value }\end{array}$} \\
\hline & $\begin{array}{l}\text { Weber } \\
\text { Class } \\
A+B \\
(n=11)\end{array}$ & $\begin{array}{l}\text { Weber } \\
\text { Class C } \\
(n=5)\end{array}$ & $\begin{array}{l}\text { Weber } \\
\text { Class A } \\
+B(n= \\
7)\end{array}$ & $\begin{array}{l}\text { Weber } \\
\text { Class C } \\
(n=5)\end{array}$ & \multirow[t]{2}{*}{$\begin{array}{l}\text { p-value } \\
(A+B)\end{array}$} & \multirow[t]{2}{*}{$\begin{array}{l}\text { p-value } \\
\text { (C) }\end{array}$} & \multirow[t]{2}{*}{$\begin{array}{l}\text { p-value } \\
\text { HFpEF }\end{array}$} & \multirow[t]{2}{*}{$\begin{array}{l}\text { p-value } \\
\text { HFrEF }\end{array}$} & \\
\hline & $\begin{array}{l}\text { Mean } \\
\pm \text { SD }\end{array}$ & $\begin{array}{l}\text { Mean } \pm \\
\text { SD }\end{array}$ & $\begin{array}{l}\text { Mean } \pm \\
\text { SD }\end{array}$ & $\begin{array}{l}\text { Mean } \pm \\
\text { SD }\end{array}$ & & & & & \\
\hline Male (n, \%) & $\begin{array}{l}10 \\
(90.9 \%)\end{array}$ & $\begin{array}{l}1 \\
(20.0 \%)\end{array}$ & $5(71.4 \%)$ & $\begin{array}{l}3 \\
(60.0 \%)\end{array}$ & - & - & $0.013^{c *}$ & $\begin{array}{l}> \\
0.999^{c}\end{array}$ & $>0.999^{c}$ \\
\hline Age, years & $\begin{array}{l}53.7 \pm \\
9.4\end{array}$ & $\begin{array}{l}59.8 \pm \\
15.7\end{array}$ & $\begin{array}{l}53.7 \pm \\
7.9\end{array}$ & $\begin{array}{l}55.4 \pm \\
7.1\end{array}$ & $0.998^{a}$ & $0.590^{\mathrm{a}}$ & $0.457^{a}$ & $0.708^{a}$ & $0.737^{a}$ \\
\hline $\begin{array}{l}\text { Body Mass } \\
\text { Index, kg/m² }\end{array}$ & $\begin{array}{l}30.0 \pm \\
3.9\end{array}$ & $\begin{array}{l}30.1 \pm \\
4.8\end{array}$ & $\begin{array}{l}28.2 \pm \\
5.5\end{array}$ & $\begin{array}{l}28.3 \pm \\
5.2\end{array}$ & $0.476^{a}$ & $0.588^{a}$ & $0.969^{a}$ & $0.981^{a}$ & $0.335^{a}$ \\
\hline \multicolumn{10}{|l|}{ Drugs } \\
\hline $\begin{array}{l}\text { Beta-blocker (n, } \\
\%)\end{array}$ & $\begin{array}{l}10 \\
(90.1 \%)\end{array}$ & $\begin{array}{l}5 \\
(100.0 \%)\end{array}$ & $\begin{array}{l}7 \\
(100.0 \%)\end{array}$ & $\begin{array}{l}5 \\
(100.0 \%)\end{array}$ & - & - & $>0.999^{c}$ & $\begin{array}{l}> \\
0.999^{c}\end{array}$ & $>0.999^{c}$ \\
\hline ACEI $(n, \%)$ & $\begin{array}{l}6 \\
(54.6 \%)\end{array}$ & $\begin{array}{l}2 \\
(40.0 \%)\end{array}$ & $6(85.7 \%)$ & $\begin{array}{l}3 \\
(60.0 \%)\end{array}$ & - & - & $>0.999^{c}$ & $\begin{array}{l}> \\
0.523^{c}\end{array}$ & $0.253^{c}$ \\
\hline $\begin{array}{l}\text { Angiotensin } \\
\text { Receptor } \\
\text { Blockers (n, \%) }\end{array}$ & $\begin{array}{l}2 \\
(18.2 \%)\end{array}$ & 2 & $3(42.9 \%)$ & $0(0.0 \%)$ & - & - & $>0.547^{c}$ & $\begin{array}{l}> \\
0.205^{c}\end{array}$ & $>0.999^{c}$ \\
\hline Diuretics (n, \%) & $\begin{array}{l}2 \\
(18.2 \%)\end{array}$ & $\begin{array}{l}3 \\
(60.0 \%)\end{array}$ & $\begin{array}{l}7 \\
(100.0 \%)\end{array}$ & $\begin{array}{l}5 \\
(100.0 \%)\end{array}$ & - & - & $>0.245^{c}$ & $\begin{array}{l}> \\
0.999^{c}\end{array}$ & $0.020^{c *}$ \\
\hline Statins (n, \%) & $\begin{array}{l}10 \\
(90.9 \%)\end{array}$ & $\begin{array}{l}3 \\
(60.0 \%)\end{array}$ & $\begin{array}{l}5 \\
(71.4 .0 \%)\end{array}$ & $\begin{array}{l}3 \\
(60.0 \%)\end{array}$ & - & - & $0.214^{\mathrm{c}}$ & $\begin{array}{l}> \\
0.999^{c}\end{array}$ & $0.418^{\mathrm{C}}$ \\
\hline $\begin{array}{l}\text { Coronary } \\
\text { Vasodilators (n, } \\
\%)\end{array}$ & $\begin{array}{l}1 \\
(9.1 \%)\end{array}$ & $\begin{array}{l}2 \\
(40.0 \%)\end{array}$ & $1(14.3 \%)$ & $\begin{array}{l}1 \\
(20.0 \%)\end{array}$ & - & - & $>0.214^{c}$ & $\begin{array}{l}> \\
0.999^{c}\end{array}$ & $>0.999^{c}$ \\
\hline $\begin{array}{l}\text { Antidiabetic (n, } \\
\%)\end{array}$ & $\begin{array}{l}2 \\
(18.2 \%)\end{array}$ & $\begin{array}{l}1 \\
(20.0 \%)\end{array}$ & $1(14.3 \%)$ & $\begin{array}{l}1 \\
(20.0 \%)\end{array}$ & - & - & $>0.999^{c}$ & $\begin{array}{l}> \\
0.999^{c}\end{array}$ & $>0.999^{c}$ \\
\hline $\begin{array}{l}\text { Anticoagulants } \\
(n, \%)\end{array}$ & $\begin{array}{l}0 \\
(0.0 \%)\end{array}$ & $0(0.0 \%)$ & $1(14.3 \%)$ & 2 & - & - & $>0.999^{c}$ & $0.523^{c}$ & $0.067^{c}$ \\
\hline \multicolumn{10}{|l|}{ Echocardiogram } \\
\hline $\begin{array}{l}\text { LVEF Simpson } \\
(n, \%)\end{array}$ & $\begin{array}{l}58.4 \pm \\
6.3\end{array}$ & $\begin{array}{l}59.2 \pm \\
6.3\end{array}$ & $\begin{array}{l}34.4 \pm \\
4.9\end{array}$ & $\begin{array}{l}28.6 \pm \\
7.4\end{array}$ & $\begin{array}{l}< \\
0.0001^{b *}\end{array}$ & $0.0001^{a *}$ & $0.811^{\mathrm{a}}$ & $0.407^{b}$ & $\begin{array}{l}< \\
0.0001^{a *}\end{array}$ \\
\hline
\end{tabular}

Legend: Values are expressed as mean \pm standard deviation (SD) or absolute and relative frequencies $n(\%)$. Statistics: ${ }^{\text {a }}$ Unpaired t-test; ${ }^{b}$ Mann-Whitney $\mathrm{U}$ test; ${ }^{\mathrm{c}}$ Fisher's Exact Test. ${ }^{*} \mathrm{p} \leq 0.05$. Abbreviations: HFpEF, heart failure with preserved ejection fraction; $\mathrm{HFrEF}$, heart failure with reduced ejection fraction; $\mathrm{kg} / \mathrm{m}^{2}$, kilogram per square meter; $\mathrm{mL} / \mathrm{m}^{2}$, millimeter per square meter; $\mathrm{g} / \mathrm{m}^{2}$, gram per square meter; $\mathrm{ACEl}$, angiotensin-converting enzyme inhibitors; LVEF, left ventricular ejection fraction; E/e', early mitral inflow velocity and mitral annular early diastolic velocity ratio; $\mathrm{cm} / \mathrm{s}$, centimeters per second; min, minute; $\mathrm{W}$, watt; $\mathrm{RER}$, respiratory gas exchange ratio; $\mathrm{VO}_{2}$, oxygen uptake; $\mathrm{mL} \cdot \mathrm{kg}^{-1} \cdot \mathrm{min}^{-1}$, millimeter per minute per kilogram; $\mathrm{ml} / \mathrm{min}$, millimeter per minute; $\mathrm{VE} / \mathrm{VCO}_{2}$, minute ventilation/carbon dioxide production slope; $\mathrm{L} / \mathrm{min}$, liters per minute. 


\begin{tabular}{|c|c|c|c|c|c|c|c|c|c|}
\hline \multirow[b]{2}{*}{$\begin{array}{l}\text { Left Atrial } \\
\text { Volume Index, } \\
\mathrm{mL} / \mathrm{m}^{2}\end{array}$} & \multicolumn{2}{|c|}{ HFpEF $(n=16)$} & \multicolumn{2}{|c|}{ HFrEF $(n=12)$} & \multicolumn{2}{|c|}{ HFpEF vs. HFrEF } & \multicolumn{2}{|c|}{$\begin{array}{l}\text { Weber Class A+B } \\
\text { vs. } \\
\text { Weber Class C }\end{array}$} & \multirow{2}{*}{$\begin{array}{l}\text { HFpEF } \\
\text { vs. } \\
\text { HFrEF } \\
< \\
0.0001^{b *}\end{array}$} \\
\hline & $\begin{array}{l}25.0 \pm \\
2.9\end{array}$ & $\begin{array}{l}29.1 \pm \\
10.4\end{array}$ & $\begin{array}{l}34.4 \pm \\
5.7\end{array}$ & $\begin{array}{l}39.4 \pm \\
4.8\end{array}$ & $0.004^{a *}$ & $0.094^{\mathrm{a}}$ & $0.435^{\mathrm{a}}$ & $0.136^{a}$ & \\
\hline $\begin{array}{l}\text { Left Ventricular } \\
\text { Mass Index, } \\
\mathrm{g} / \mathrm{m}^{2}\end{array}$ & $\begin{array}{l}83.8 \pm \\
10.8\end{array}$ & $\begin{array}{l}95.4 \pm \\
38.4\end{array}$ & $\begin{array}{l}102.6 \pm \\
33.7\end{array}$ & $\begin{array}{l}141.0 \pm \\
22.9\end{array}$ & $0.197^{a}$ & $0.059^{a}$ & $0.543^{\mathrm{a}}$ & $0.041^{a^{*}}$ & $0.002^{b \star}$ \\
\hline $\mathrm{E} / \mathrm{e}^{\prime}, \mathrm{cm} / \mathrm{s}$ & $\begin{array}{l}6.6 \pm \\
2.1\end{array}$ & $7.5 \pm 0.9$ & $\begin{array}{l}10.9 \pm \\
4.6\end{array}$ & $\begin{array}{l}11.9 \pm \\
4.7\end{array}$ & $0.050^{a *}$ & $0.099^{a}$ & $0.273^{\mathrm{a}}$ & $0.706^{a}$ & $0.006^{\mathrm{a} *}$ \\
\hline $\begin{array}{l}\text { Mean e' (septal } \\
\text { wall), cm/s }\end{array}$ & $\begin{array}{l}8.0 \pm \\
1.9\end{array}$ & $6.4 \pm 1.7$ & $5.1 \pm 1.3$ & $3.8 \pm 0.8$ & $0.003^{b *}$ & $0.022^{a *}$ & $0.127^{a}$ & $0.096^{a}$ & $\begin{array}{l}<.0001^{\mathrm{a} *} \\
\end{array}$ \\
\hline $\begin{array}{l}\text { Mean e' (lateral } \\
\text { wall), } \mathrm{cm} / \mathrm{s}\end{array}$ & $\begin{array}{l}12.6 \pm \\
3.7\end{array}$ & $9.4 \pm 3.1$ & $8.3 \pm 1.9$ & $5.2 \pm 1.8$ & $0.005^{a *}$ & $0.040^{b \star}$ & $0.097^{a}$ & $0.032^{b *}$ & $0.001^{a \star}$ \\
\hline $\begin{array}{l}\text { Cardiopulmonary } \\
\text { exercise testing }\end{array}$ & $(n=11)$ & $(n=5)$ & $(n=7)$ & $(n=5)$ & & & & & \\
\hline Exercise, min & $\begin{array}{l}10.8 \pm \\
2.5\end{array}$ & $6.6 \pm 0.9$ & $\begin{array}{l}10.1 \pm \\
2.6\end{array}$ & $6.9 \pm 2.2$ & $0.641^{b}$ & $0.782^{\mathrm{a}}$ & $0.001^{b *}$ & $0.048^{a}$ & $0.639^{b}$ \\
\hline Peak RER & $\begin{array}{l}1.2 \pm \\
0.1\end{array}$ & $1.3 \pm 0.1$ & $1.2 \pm 0.1$ & $1.3 \pm 0.1$ & $0.643^{b}$ & $>0.999^{a}$ & $0.179^{b}$ & $0.191^{\mathrm{a}}$ & $0.859^{b}$ \\
\hline $\begin{array}{l}\text { Peak Power } \\
\text { Output, W }\end{array}$ & $\begin{array}{l}140.3 \\
\pm 27.0\end{array}$ & $\begin{array}{l}84.8 \pm \\
15.3\end{array}$ & $\begin{array}{l}107.3 \pm \\
26.0\end{array}$ & $\begin{array}{l}74.8 \pm \\
21.7\end{array}$ & $0.024^{a *}$ & $0.426^{\mathrm{a}}$ & $0.0002^{a \star}$ & $0.041^{a *}$ & $0.024^{a \star}$ \\
\hline Peak FC, bpm & $\begin{array}{l}141.9 \\
\pm 18.0\end{array}$ & $\begin{array}{l}116.8 \pm \\
28.3\end{array}$ & $\begin{array}{l}130.6 \pm \\
16.5\end{array}$ & $\begin{array}{l}125.2 \pm \\
25.6\end{array}$ & $0.192^{\mathrm{a}}$ & $0.605^{a}$ & $0.113^{\mathrm{a}}$ & $0.694^{a}$ & $0.516^{a}$ \\
\hline $\begin{array}{l}\text { Peak } \mathrm{VO}_{2} \\
\mathrm{ml} \cdot \mathrm{kg}^{-1} \cdot \mathrm{min}^{-1}\end{array}$ & $\begin{array}{l}22.1 \pm \\
3.4\end{array}$ & $\begin{array}{l}14.4 \pm \\
1.2\end{array}$ & $\begin{array}{l}19.2 \pm \\
2.7\end{array}$ & $\begin{array}{l}13.7 \pm \\
1.6\end{array}$ & $0.060^{\mathrm{a}}$ & $0.434^{\mathrm{a}}$ & $\begin{array}{l}< \\
0.0001^{a *}\end{array}$ & $0.001^{a *}$ & $0.081^{a}$ \\
\hline $\begin{array}{l}\% \text { Predicted peak } \\
\mathrm{VO}_{2} \\
\mathrm{ml} \cdot \mathrm{kg}^{-1} \cdot \mathrm{min}^{-1}\end{array}$ & $\begin{array}{l}66.0 \pm \\
9.1\end{array}$ & $\begin{array}{l}54.3 \pm \\
17.1\end{array}$ & $\begin{array}{l}58.8 \pm \\
10.7\end{array}$ & $\begin{array}{l}43.4 \pm \\
7.5\end{array}$ & $0.167^{a}$ & $0.151^{\mathrm{b}}$ & $0.052^{b}$ & $0.048^{b *}$ & $0.046^{a *}$ \\
\hline $\begin{array}{l}\text { Peak } \mathrm{VO}_{2} \\
\mathrm{ml} \cdot \mathrm{min}^{-1}\end{array}$ & $\begin{array}{l}1884.6 \\
\pm 312.7\end{array}$ & $\begin{array}{l}1180.6 \\
\pm 126.2\end{array}$ & $\begin{array}{l}1469.6 \pm \\
344.0\end{array}$ & $\begin{array}{l}1034.8 \\
\pm 251.5\end{array}$ & $0.024^{a *}$ & $0.291^{\mathrm{a}}$ & $\begin{array}{l}<.0001^{a *} \\
\end{array}$ & $0.030^{a *}$ & $0.020^{a \star}$ \\
\hline $\begin{array}{l}\mathrm{VE} / \mathrm{VCO}_{2} \text { Slope, } \\
\mathrm{L} / \mathrm{min}\end{array}$ & $\begin{array}{l}27.9 \pm \\
3.7\end{array}$ & $\begin{array}{l}28.8 \pm \\
8.0\end{array}$ & $\begin{array}{l}31.1 \pm \\
5.4\end{array}$ & $\begin{array}{l}30.1 \pm \\
4.0\end{array}$ & $0.196^{\mathrm{a}}$ & $0.310^{\mathrm{b}}$ & $0.510^{\mathrm{b}}$ & $0.876^{\mathrm{b}}$ & $0.084^{b}$ \\
\hline
\end{tabular}

Legend: Values are expressed as mean \pm standard deviation (SD) or absolute and relative frequencies $n$ (\%). Statistics: ${ }^{\text {a }}$ Unpaired t-test; ${ }^{b}$ Mann-Whitney $\mathrm{U}$ test; ${ }^{\mathrm{c}}$ Fisher's Exact Test. ${ }^{*} \mathrm{p} \leq 0.05$. Abbreviations: HFpEF, heart failure with preserved ejection fraction; $\mathrm{HFrEF}$, heart failure with reduced ejection fraction; $\mathrm{kg} / \mathrm{m}^{2}$, kilogram per square meter; $\mathrm{mL} / \mathrm{m}^{2}$, millimeter per square meter; $\mathrm{g} / \mathrm{m}^{2}$, gram per square meter; $\mathrm{ACEl}$, angiotensin-converting enzyme inhibitors; LVEF, left ventricular ejection fraction; E/e', early mitral inflow velocity and mitral annular early diastolic velocity ratio; $\mathrm{cm} / \mathrm{s}$, centimeters per second; min, minute; $\mathrm{W}$, watt; $\mathrm{RER}$, respiratory gas exchange ratio; $\mathrm{VO}_{2}$, oxygen uptake; $\mathrm{mL} \cdot \mathrm{kg}^{-1} \cdot \mathrm{min}^{-1}$, millimeter per minute per kilogram; $\mathrm{ml} / \mathrm{min}$, millimeter per minute; $\mathrm{VE} / \mathrm{VCO}_{2}$, minute ventilation/carbon dioxide production slope; $\mathrm{L} / \mathrm{min}$, liters per minute.

\section{Peripheral muscle microcirculation dynamics during isokinetic muscle strength testing}

Altogether, within HFpEF, Weber Class $\mathrm{C}$ patients presented a poor capability to reach a greater oxygen extraction during exercise than Classes A and B. Among those with Weber $\mathrm{C}$ severity, while HFrEF patients reached lower oxyhemoglobin $\left(\mathrm{O}_{2} \mathrm{Hb}, \mu \mathrm{M}\right)(-10.9 \pm$ 3.8 vs. $-23.7 \pm 5.7, p=0.029)$ during exercise, $\mathrm{HFpEF}$ kept lower $\mathrm{O}_{2} \mathrm{Hb}$ during the recovery period $(-3.0 \pm 3.4$ vs. $5.9 \pm 2.8, p=0.007)$ 
(Table 2). During the isokinetic muscle strength test, a trend of higher TSI (\%) for HFpEF was observed, as compared to HFrEF patients with Class $A+B$ severity $(44.2 \pm 8.2$ vs. $36.0 \pm 2.4, p=0.060)$ (Table 2$)$.

Baseline tissue saturation index $(\mathrm{TSI}, \%)$, oxyhemoglobin $\left(\mathrm{O}_{2} \mathrm{Hb}, \mu \mathrm{M}\right)$, and deoxyhemoglobin $(\mathrm{HHb}, \mu \mathrm{M})$ were similar between heart failure phenotypes (HFrEF and HFpEF) with different severity classifications (Weber Class A+B vs. C) (Table 2; $p>0.05$ ).

However, significant differences for $\mathrm{HHb}(\mu \mathrm{M})$ parameter were observed during the recovery phase when comparing both phenotypes within Weber Class A + B. This indicated a higher value in the HFrEF group $(18.8 \pm 4.8$ vs. $8.9 \pm 5.6, p=0.042)$, which means that HFrEF presented a worse recovery than HFpEF. The logic is that HFrEF presents better $\mathrm{O}_{2}$ extraction during strength exercise than HFpEF, which is probably why HFrEF recovery is worse than HFpEF.

Lastly, we did not observe significant differences for $\mathrm{HHb}(\mu \mathrm{M})$ during the exercise phase between both phenotypes and clinical severities $(p>0.05)$ (Table 2). However, significant differences for $\mathrm{HHb}(\mu \mathrm{M})$ parameter were observed during the recovery phase when comparing both phenotypes within Weber Class A + B. This indicated a higher value in the HFrEF group $(18.8 \pm 4.8 \mathrm{vs} .8 .9 \pm$ $5.6, p=0.042)$.

The TSI (Fig. 1) recovery period was longer in the HFpEF compared to the HFrEF group in both Weber A+B and Weber $C$ severity subgroups. Similarly, the recovery period in TSI was significantly longer in Weber A+B than C patients in both HFpEF and HFrEF phenotypes (Fig. 1).

Although the drop in $\mathrm{O}_{2} \mathrm{Hb}$ during exercise was similar in both phenotypes when considering the severity $\mathrm{A}+\mathrm{B}$, the return to baseline was faster in HFrEF than HFpEF (Fig. 2). This faster return to baseline threshold in HFrEF also occurred when comparing both phenotypes at Weber $C$ severity. In addition, when comparing the severities $A+B$ versus $C$ in HFpEF, a higher drop-out occurred at Weber Class $A+B$, and a worse recovery was identified for the patients with Weber $C$ severity.

The recovery period for $\mathrm{HHb}$ (Fig. 3) was extended for HFpEF compared to HFrEF severity A + B. Similarly, in the first seconds of recovery for severity $\mathrm{C}, \mathrm{HFpEF}$ maintained more $\mathrm{HHb}$, while HFrEF dropped out faster. HFpEF had a worse recovery compared to $\mathrm{HFrEF}$, regardless of severity. 
Table 2

NIRS during isokinetic muscle strength parameters in both heart failure phenotypes and Weber Class.

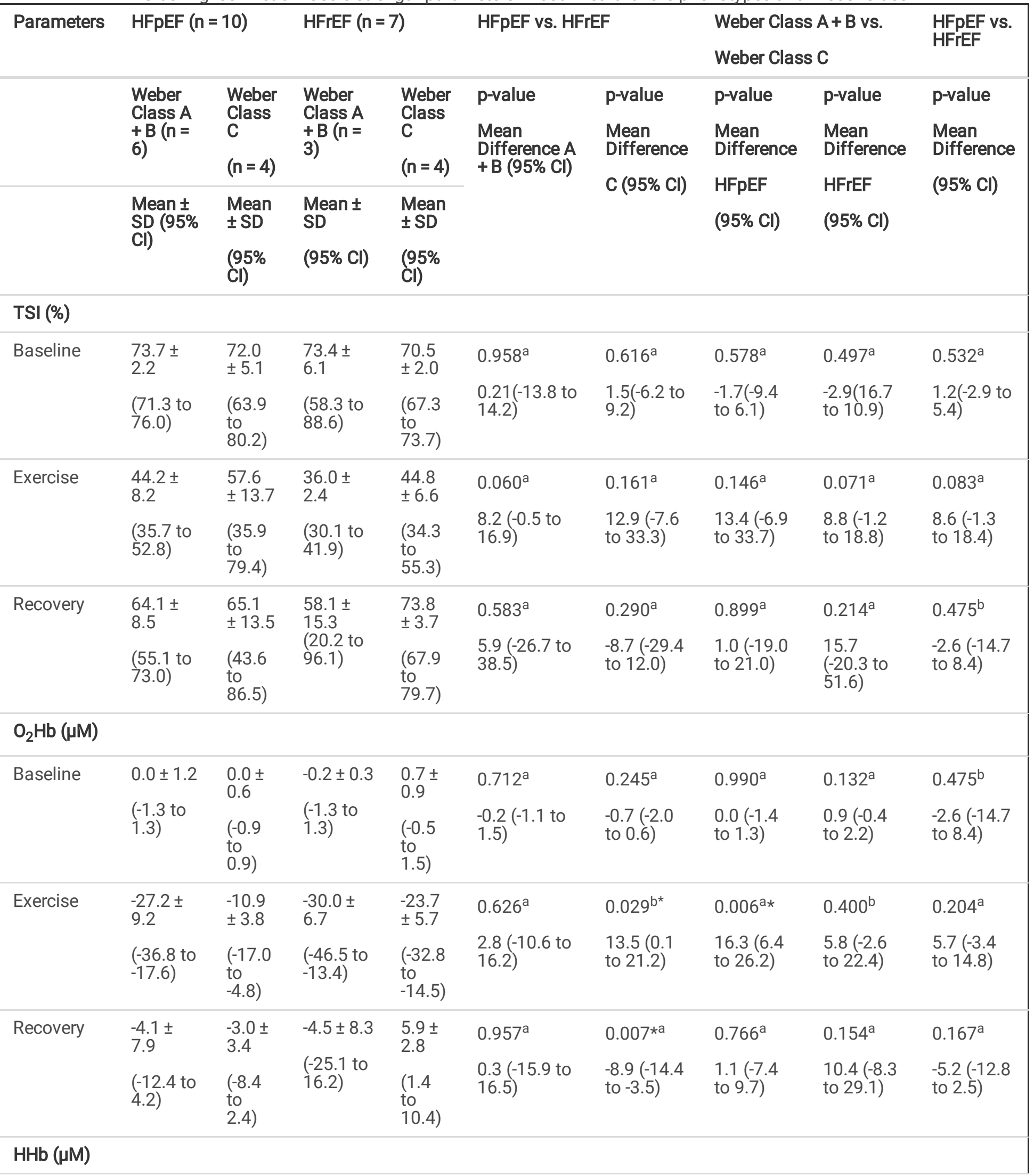

Legend: Values are expressed as mean \pm standard deviation (SD), mean difference (MD), and 95\% confidence interval (95\% Cl) or median difference, number of included patients (n). Statistics: ${ }^{a}$ Unpaired t-test (MD and $\left.95 \% \mathrm{Cl}\right)$; ${ }^{b}$ Mann-Whitney test (Hodge-Lehmann's median difference considered); ${ }^{*} \mathrm{p} \leq 0.05$. Abbreviations: HFpEF, heart failure with preserved ejection fraction; HFrEF, heart failure with reduced ejection fraction. 


\begin{tabular}{|c|c|c|c|c|c|c|c|c|c|}
\hline \multirow{2}{*}{$\begin{array}{l}\text { Parameters } \\
\text { Baseline }\end{array}$} & \multicolumn{2}{|c|}{ HFpEF $(n=10)$} & \multicolumn{2}{|c|}{ HFrEF $(n=7)$} & \multicolumn{2}{|c|}{ HFpEF vs. HFrEF } & \multicolumn{2}{|c|}{$\begin{array}{l}\text { Weber Class A+B vs. } \\
\text { Weber Class C }\end{array}$} & \multirow{2}{*}{$\begin{array}{l}\text { HFpEF vs. } \\
\text { HFrEF } \\
0.124^{a}\end{array}$} \\
\hline & $\begin{array}{l}-0.1 \pm \\
0.3\end{array}$ & $\begin{array}{l}-0.2 \pm \\
0.4\end{array}$ & $-0.1 \pm 0.2$ & $\begin{array}{l}0.5 \pm \\
0.6\end{array}$ & $0.809^{a}$ & $0.128^{a}$ & $0.866^{a}$ & $0.160^{\mathrm{a}}$ & \\
\hline & $\begin{array}{l}(-0.4 \text { to } \\
0.2)\end{array}$ & $\begin{array}{l}(-0.7 \\
\text { to } \\
0.4)\end{array}$ & & $\begin{array}{l}(-0.5 \\
\text { to } \\
1.0)\end{array}$ & & & & & \\
\hline & & & $\begin{array}{l}(-0.6 \text { to } \\
0.5)\end{array}$ & & $\begin{array}{l}-0.04(-0.5 \text { to } \\
0.4)\end{array}$ & $\begin{array}{l}-0.6(-1.5 \\
\text { to } 0.3)\end{array}$ & $\begin{array}{l}0.0(-0.6 \\
\text { to } 0.5)\end{array}$ & $\begin{array}{l}0.5(-0.3 \\
\text { to } 1.4)\end{array}$ & $\begin{array}{l}-0.4(-0.9 \\
\text { to } 0.1)\end{array}$ \\
\hline \multirow[t]{2}{*}{ Exercise } & $\begin{array}{l}14.0 \pm \\
6.4\end{array}$ & $\begin{array}{l}3.4 \pm \\
7.6\end{array}$ & $\begin{array}{l}11.6 \pm \\
5.2\end{array}$ & $\begin{array}{l}8.3 \pm \\
4.3\end{array}$ & $0.571^{a}$ & $0.313^{\mathrm{a}}$ & $0.062^{a}$ & $0.415^{\mathrm{a}}$ & $0.992^{\mathrm{a}}$ \\
\hline & $\begin{array}{l}(7.4 \text { to } \\
20.7)\end{array}$ & $\begin{array}{l}(-8.7 \\
\text { to } \\
15.5)\end{array}$ & $\begin{array}{l}(-1.2 \text { to } \\
24.5)\end{array}$ & $\begin{array}{l}(1.4 \\
\text { to } \\
15.2)\end{array}$ & $\begin{array}{l}2.4(-7.7 \text { to } \\
12.5)\end{array}$ & $\begin{array}{l}-4.9(-16.4 \\
\text { to } 6.5)\end{array}$ & $\begin{array}{l}-10.7 \\
(-22.1 \text { to } \\
0.7)\end{array}$ & $\begin{array}{l}-3.4(-13.6 \\
\text { to } 6.9)\end{array}$ & $\begin{array}{l}0.0(-6.8 \\
\text { to } 6.9)\end{array}$ \\
\hline \multirow[t]{2}{*}{ Recovery } & $8.9 \pm 5.6$ & $\begin{array}{l}4.6 \pm \\
6.1\end{array}$ & $\begin{array}{l}18.8 \pm \\
4.8\end{array}$ & $\begin{array}{l}0.7 \pm \\
1.7\end{array}$ & $0.042^{a^{*}}$ & $0.289^{a}$ & $0.313^{a}$ & $0.016^{a^{*}}$ & $0.771^{\mathrm{a}}$ \\
\hline & $\begin{array}{l}(3.0 \text { to } \\
14.8)\end{array}$ & $\begin{array}{l}(-5.0 \\
\text { to } \\
14.4)\end{array}$ & $\begin{array}{l}(6.9 \text { to } \\
30.8)\end{array}$ & $\begin{array}{l}(-2.0 \\
\text { to } \\
3.5)\end{array}$ & $\begin{array}{l}-10.0(-19.4 \\
\text { to }-0.5)\end{array}$ & $\begin{array}{l}4.0(-5.4 \\
\text { to } 13.3)\end{array}$ & $\begin{array}{l}-4.2(-13.5 \\
\text { to } 5.1)\end{array}$ & $\begin{array}{l}-18.1 \\
(-28.9 \text { to } \\
-7.3)\end{array}$ & $\begin{array}{l}-1.3(-11.0 \\
\text { to } 8.4)\end{array}$ \\
\hline
\end{tabular}

\section{Isokinetic muscle strength parameters}

No isokinetic muscle strength parameters (peak torque, peak torque/body mass, maximal repetition total work, work/body weight, total work, work fatigue, and average power) were different among phenotypes, nor between disease severity states $(p>0.05)$ (Table 3).

\section{Ultrasound-derived parameters (echo intensity and muscle thickness)}

Muscle thickness (MT, cm) (Table 4) did not exhibit significant differences between HFpEF and HFrEF groups for quadriceps femoris and rectus femoris analysis $(p>0.05)$. In addition, smaller MT was observed in HFpEF patients with Weber Class $C(p=$ 0.02). There was no difference in echo intensity of the rectus femoris between phenotypes $(p>0.05)$ despite the greater echo intensity on the HFpEF on Weber C compared to the Weber Class A + B. $(31.7 \pm 9.0$ vs. $14.1 \pm 8.7, p=0.003)$.

\section{Associations}

Regarding the association between muscle microcirculation dynamics during strength exercise testing $\left(\mathrm{TSI}, \mathrm{O}_{2} \mathrm{Hb}\right.$, and $\left.\mathrm{HHb}\right)$ and isokinetic muscle strength parameters (PT) for both phenotypes and severities, there was no statistical significance for any association $(p>0.05)$ (Table 5). There was also no correlation between TSI, $\mathrm{O}_{2} \mathrm{Hb}$, and $\mathrm{HHb}$ with peak $\mathrm{VO}_{2}\left(\mathrm{~mL} \mathrm{~kg}^{-1} \cdot \mathrm{min}^{-1}\right)(p>$ 0.05). The association between the muscle microcirculation dynamics $\left(\mathrm{TSI}, \mathrm{O}_{2} \mathrm{Hb}\right.$, and $\mathrm{HHb}$ ) with peak power output (W) were not found $(p>0.05)$.

However, regarding the associations between ultrasound-derived measures of rectus femoris (RF) echo intensity and muscle thickness $\left(\mathrm{MT}, \mathrm{cm}\right.$ ) and cardiorespiratory fitness (peak $\mathrm{VO}_{2}, \mathrm{~mL} \cdot \mathrm{kg}^{-1} \cdot \mathrm{min}^{-1}$ ), RF_El was strongly negatively associated with peak $\mathrm{VO}_{2}\left(\mathrm{~mL} \cdot \mathrm{kg}^{-1} \cdot \mathrm{min}^{-1}\right)$ only in HFpEF phenotype in patients with worse severity status (Weber $\left.\mathrm{C}\right)(\mathrm{p}=0.032 ; \mathrm{r}=-0.910)$. A moderate association between RF_MT and isokinetic PT was observed only in HFrEF phenotype with Weber $C(p=0.049 ; r=-0.880)$. There 
was also no correlation between RF_El with PT $(\mathrm{Nm})(\mathrm{p}>0.05)$. Likewise, there was no association between RF_MT and peak VO 2 or peak power output $(p>0.05)$. 
Table 3

Isokinetic muscle strength parameters in both heart failure phenotypes and Weber Class.

\begin{tabular}{|c|c|c|c|c|c|c|c|c|c|}
\hline \multirow[t]{7}{*}{ Parameters } & \multicolumn{2}{|c|}{ HFpEF $(n=10)$} & \multicolumn{2}{|c|}{ HFrEF $(n=7)$} & \multicolumn{2}{|c|}{ HFpEF vs. HFrEF } & \multicolumn{2}{|c|}{$\begin{array}{l}\text { Weber Class A+ B vs. } \\
\text { Weber Class C }\end{array}$} & $\begin{array}{l}\text { HFpEF vs. } \\
\text { HFrEF }\end{array}$ \\
\hline & \multirow{4}{*}{$\begin{array}{l}\text { Weber } \\
\text { Class A } \\
+B(n= \\
6)\end{array}$} & \multirow{4}{*}{$\begin{array}{l}\text { Weber } \\
\text { Class C } \\
(n=4)\end{array}$} & \multirow{4}{*}{$\begin{array}{l}\text { Weber } \\
\text { Class A } \\
+B(n= \\
3)\end{array}$} & \multirow{4}{*}{$\begin{array}{l}\text { Weber } \\
\text { Class C } \\
(n=4)\end{array}$} & \multirow{4}{*}{$\begin{array}{l}\text { p-value } \\
\text { Mean } \\
\text { Difference } \\
\text { A+B (95\% } \\
\text { Cl) }\end{array}$} & \multirow{4}{*}{$\begin{array}{l}\text { p-value } \\
\text { Mean } \\
\text { Difference } \\
\text { C }(95 \% \mathrm{Cl})\end{array}$} & p-value & p-value & p-value \\
\hline & & & & & & & $\begin{array}{l}\text { Mean } \\
\text { Difference }\end{array}$ & $\begin{array}{l}\text { Mean } \\
\text { Difference }\end{array}$ & $\begin{array}{l}\text { Mean } \\
\text { Difference }\end{array}$ \\
\hline & & & & & & & HFpEF & HFrEF & $(95 \% \mathrm{Cl})$ \\
\hline & & & & & & & $(95 \% \mathrm{Cl})$ & $(95 \% \mathrm{Cl})$ & \\
\hline & $\begin{array}{l}\text { Mean } \pm \\
\text { SD }\end{array}$ & $\begin{array}{l}\text { Mean } \pm \\
\text { SD }\end{array}$ & $\begin{array}{l}\text { Mean } \pm \\
\text { SD }\end{array}$ & $\begin{array}{l}\text { Mean } \pm \\
\text { SD }\end{array}$ & & & & & \\
\hline & $\begin{array}{l}(95 \% \\
\mathrm{Cl})\end{array}$ & $\begin{array}{l}(95 \% \\
\mathrm{Cl})\end{array}$ & $\begin{array}{l}(95 \% \\
\mathrm{Cl})\end{array}$ & $\begin{array}{l}(95 \% \\
\mathrm{Cl})\end{array}$ & & & & & \\
\hline \multirow[t]{2}{*}{ PT, Nm } & $\begin{array}{l}125.5 \pm \\
25.7\end{array}$ & $\begin{array}{l}68.2 \pm \\
10.8\end{array}$ & $\begin{array}{l}136.8 \pm \\
47.5\end{array}$ & $\begin{array}{l}83.4 \pm \\
38.8\end{array}$ & $>0.999^{b}$ & $0.500^{\mathrm{a}}$ & $0.019 * b$ & $0.190^{\mathrm{a}}$ & $0.887^{b}$ \\
\hline & $\begin{array}{l}\text { (98.6 to } \\
152.4)\end{array}$ & $\begin{array}{l}(51.1 \text { to } \\
85.3)\end{array}$ & $\begin{array}{l}(18.7 \text { to } \\
254.8)\end{array}$ & $\begin{array}{l}(21.6 \text { to } \\
145.1)\end{array}$ & $\begin{array}{l}-2.0(-74.4 \\
\text { to } 47.6)\end{array}$ & $\begin{array}{l}-15.2 \\
(-74.7 \text { to } \\
44.4)\end{array}$ & $\begin{array}{l}64.8(11.4 \\
\text { to } 83.6)\end{array}$ & $\begin{array}{l}53.4 \\
(-41.3 \text { to } \\
148.1)\end{array}$ & $\begin{array}{l}10.4 \\
(-49.8 \text { to } \\
37.2)\end{array}$ \\
\hline \multirow{2}{*}{$\begin{array}{l}\text { PT/Body } \\
\text { Mass, } \\
\text { Nm.kg }\end{array}$} & $\begin{array}{l}139.2 \pm \\
28.9\end{array}$ & $\begin{array}{l}82.4 \pm \\
1.9\end{array}$ & $\begin{array}{l}159.7 \pm \\
32.6\end{array}$ & $\begin{array}{l}105.2 \pm \\
37.9\end{array}$ & \multirow{2}{*}{$\begin{array}{l}0.414^{a} \\
-20.5 \\
(-84.6 \text { to } \\
43.7)\end{array}$} & \multirow{2}{*}{$\begin{array}{l}0.314^{\mathrm{a}} \\
-22.9 \\
(-83.0 \text { to } \\
37.3)\end{array}$} & \multirow{2}{*}{$\begin{array}{l}0.005^{\star a} \\
56.8(26.5 \\
\text { to } 87.2)\end{array}$} & \multirow{2}{*}{$\begin{array}{l}0.099^{a} \\
54.4 \\
(-15.1 \text { to } \\
123.9)\end{array}$} & \multirow{2}{*}{$\begin{array}{l}0.561^{\mathrm{a}} \\
-12.1 \\
(-56.3 \text { to } \\
32.1)\end{array}$} \\
\hline & $\begin{array}{l}(108.9 \\
\text { to } \\
169.6)\end{array}$ & $\begin{array}{l}\text { (79.4 to } \\
85.3)\end{array}$ & $\begin{array}{l}\text { (78.6 to } \\
240.7)\end{array}$ & $\begin{array}{l}(45.0 \text { to } \\
165.5)\end{array}$ & & & & & \\
\hline \multirow{2}{*}{$\begin{array}{l}\text { Maximal } \\
\text { Repetition } \\
\text { Total Work, } \\
\text { J }\end{array}$} & $\begin{array}{l}143.5 \pm \\
32.2\end{array}$ & $\begin{array}{l}79.5 \pm \\
15.7\end{array}$ & $\begin{array}{l}151.5 \pm \\
43.9\end{array}$ & $\begin{array}{l}99.4 \pm \\
45.2\end{array}$ & \multirow{2}{*}{$\begin{array}{l}0.796^{\mathrm{a}} \\
-8.0(-96.7 \\
\text { to } 80.7)\end{array}$} & \multirow{2}{*}{$\begin{array}{l}0.454^{\mathrm{a}} \\
-20.0 \\
(-88.5 \text { to } \\
48.6)\end{array}$} & \multirow{2}{*}{$\begin{array}{l}0.003^{* a} \\
64.1(28.4 \\
\text { to } 99.7)\end{array}$} & \multirow{2}{*}{$\begin{array}{l}0.191^{\mathrm{a}} \\
52.1 \\
(-37.9 \text { to } \\
142.1)\end{array}$} & $0.869^{a}$ \\
\hline & $\begin{array}{l}(109.7 \\
\text { to } \\
177.3)\end{array}$ & $\begin{array}{l}\text { (54.4 to } \\
104.5)\end{array}$ & $\begin{array}{l}(42.6 \text { to } \\
260.5)\end{array}$ & $\begin{array}{l}\text { (27.5 to } \\
171.4)\end{array}$ & & & & & $\begin{array}{l}-3.9(-53.9 \\
\text { to } 46.2)\end{array}$ \\
\hline \multirow[t]{2}{*}{$\begin{array}{l}\text { Work/Body } \\
\text { Weight, \% }\end{array}$} & $\begin{array}{l}159.2 \pm \\
36.6\end{array}$ & $\begin{array}{l}95.9 \pm \\
10.5\end{array}$ & $\begin{array}{l}177.9 \pm \\
26.3\end{array}$ & $\begin{array}{l}125.1 \pm \\
40.9\end{array}$ & \multirow{2}{*}{$\begin{array}{l}0.417^{a} \\
-18.7 \\
(-71.7 \text { to } \\
34.3)\end{array}$} & \multirow{2}{*}{$\begin{array}{l}0.251^{\mathrm{a}} \\
-29.2 \\
(-92.1 \text { to } \\
33.8)\end{array}$} & \multirow{2}{*}{$\begin{array}{l}0.007^{* a} \\
63.4(24.8 \\
\text { to } 101.9)\end{array}$} & \multirow{2}{*}{$\begin{array}{l}0.093^{\star a} \\
52.9 \\
(-12.8 \text { to } \\
118.5)\end{array}$} & $0.527^{a}$ \\
\hline & $\begin{array}{l}(120.8 \\
\text { to } \\
197.7)\end{array}$ & $\begin{array}{l}\text { (79.1 to } \\
112.6)\end{array}$ & $\begin{array}{l}(112.5 \\
\text { to } \\
243.3)\end{array}$ & $\begin{array}{l}(60.0 \text { to } \\
190.1)\end{array}$ & & & & & $\begin{array}{l}-13.8 \\
(-59.7 \text { to } \\
32.1)\end{array}$ \\
\hline \multirow[t]{2}{*}{$\begin{array}{l}\text { Total Work, } \\
\text { J }\end{array}$} & $\begin{array}{l}2253.5 \\
\pm 523.0\end{array}$ & $\begin{array}{l}1245.4 \\
\pm 193.5\end{array}$ & $\begin{array}{l}2351.1 \\
\pm 674.5\end{array}$ & $\begin{array}{l}1398.2 \\
\pm 593.7\end{array}$ & \multirow{2}{*}{$\begin{array}{l}0.839^{a} \\
-98.7 \\
(-1448.2 \text { to } \\
1252.8)\end{array}$} & \multirow{2}{*}{$\begin{array}{l}0.653^{a} \\
-152.9 \\
(-1055.7 \\
\text { to } 749.9)\end{array}$} & \multirow{2}{*}{$\begin{array}{l}0.004^{\star a} \\
1008.1 \\
(450.3 \text { to } \\
1565.9)\end{array}$} & \multirow{2}{*}{$\begin{array}{l}0.122^{\mathrm{a}} \\
952.9 \\
(-396.0 \text { to } \\
2301.8)\end{array}$} & \multirow{2}{*}{$\begin{array}{l}0.905^{\mathrm{a}} \\
43.6 \\
(-736.2 \text { to } \\
823.4)\end{array}$} \\
\hline & $\begin{array}{l}(1704.6 \\
\text { to } \\
2802.3)\end{array}$ & $\begin{array}{l}(937.5 \\
\text { to } \\
1553.2)\end{array}$ & $\begin{array}{l}(675.7 \\
\text { to } \\
4026.6)\end{array}$ & $\begin{array}{l}(453.5 \\
\text { to } \\
2342.9)\end{array}$ & & & & & \\
\hline $\begin{array}{l}\text { Work } \\
\text { Fatigue, \% }\end{array}$ & $\begin{array}{l}36.5 \pm \\
11.0\end{array}$ & $\begin{array}{l}32.8 \pm \\
19.2\end{array}$ & $\begin{array}{l}40.0 \pm \\
9.3\end{array}$ & $\begin{array}{l}48.1 \pm \\
2.0\end{array}$ & $0.634^{a}$ & $0.209^{a}$ & $0.747^{a}$ & $0.267^{a}$ & $0.133^{b}$ \\
\hline & $\begin{array}{l}(24.9 \text { to } \\
48.0)\end{array}$ & $\begin{array}{l}(2.2 \text { to } \\
63.4)\end{array}$ & $\begin{array}{l}(17.0 \text { to } \\
63.0)\end{array}$ & $\begin{array}{l}(44.9 \text { to } \\
51.3)\end{array}$ & $\begin{array}{l}-3.6(-21.7 \\
\text { to } 14.6)\end{array}$ & $\begin{array}{l}-15.3 \\
(-45.7 \text { to } \\
15.1)\end{array}$ & $\begin{array}{l}3.7(-24.9 \\
\text { to } 32.2)\end{array}$ & $\begin{array}{l}-8.1(-30.1 \\
\text { to } 13.9)\end{array}$ & $\begin{array}{l}-8.3(-15.1 \\
\text { to } 1.7)\end{array}$ \\
\hline
\end{tabular}

Legend: Values are expressed as mean \pm standard deviation (SD), mean difference (MD) and $95 \%$ confidence interval (95\% Cl) or median difference, number of included patients (n). Statistics: ${ }^{a}$ Unpaired t-test (MD and $95 \% \mathrm{Cl}$ ); ${ }^{\mathrm{b}}$ Mann-Whitney test (Hodge-Lehmann's median difference considered); ${ }^{*} \mathrm{p} \leq 0.05$. Abbreviations: HFpEF, heart failure with preserved ejection fraction; HFrEF, heart failure with reduced ejection fraction; PT, peak torque to Nm, newton-meter; J, Joules; W, Watt. 


\begin{tabular}{|c|c|c|c|c|c|c|c|c|c|}
\hline \multirow[t]{2}{*}{$\begin{array}{l}\text { Average } \\
\text { Power, W }\end{array}$} & $\begin{array}{l}189.4 \pm \\
47.1\end{array}$ & $\begin{array}{l}103.0 \pm \\
22.7\end{array}$ & $\begin{array}{l}206.4 \pm \\
78.1\end{array}$ & $\begin{array}{l}114.4 \pm \\
54.6\end{array}$ & $0.753^{a}$ & $0.719^{a}$ & $0.005^{\star a}$ & $0.168^{a}$ & $0.978^{a}$ \\
\hline & $\begin{array}{l}(140.0 \\
\text { to } \\
238.8)\end{array}$ & $\begin{array}{l}\text { (66.9 to } \\
139.1)\end{array}$ & $\begin{array}{l}\text { (12.4 to } \\
400.3)\end{array}$ & $\begin{array}{l}\text { (27.6 to } \\
201.2)\end{array}$ & $\begin{array}{l}-17.0 \\
(-181.1 \text { to } \\
147.1)\end{array}$ & $\begin{array}{l}-11.4 \\
(-93.4 \text { to } \\
70.6)\end{array}$ & $\begin{array}{l}86.4(34.4 \\
\text { to } 138.4)\end{array}$ & $\begin{array}{l}92.0 \\
(-64.6 \text { to } \\
248.4)\end{array}$ & $\begin{array}{l}1.0(-75.2 \\
\text { to } 77.2)\end{array}$ \\
\hline
\end{tabular}

Legend: Values are expressed as mean \pm standard deviation (SD), mean difference (MD) and 95\% confidence interval (95\% Cl) or median difference, number of included patients (n). Statistics: ${ }^{a}$ Unpaired t-test (MD and $\left.95 \% \mathrm{Cl}\right)$; ${ }^{\mathrm{b}} \mathrm{Mann}$-Whitney test (Hodge-Lehmann's median difference considered); ${ }^{\star} \mathrm{p} \leq 0.05$. Abbreviations: HFpEF, heart failure with preserved ejection fraction; HFrEF, heart failure with reduced ejection fraction; PT, peak torque to Nm, newton-meter; J, Joules; W, Watt.

Table 4

Ultrasound-derived parameters (echo intensity and muscle thickness) in both heart failure phenotypes and Weber Class.

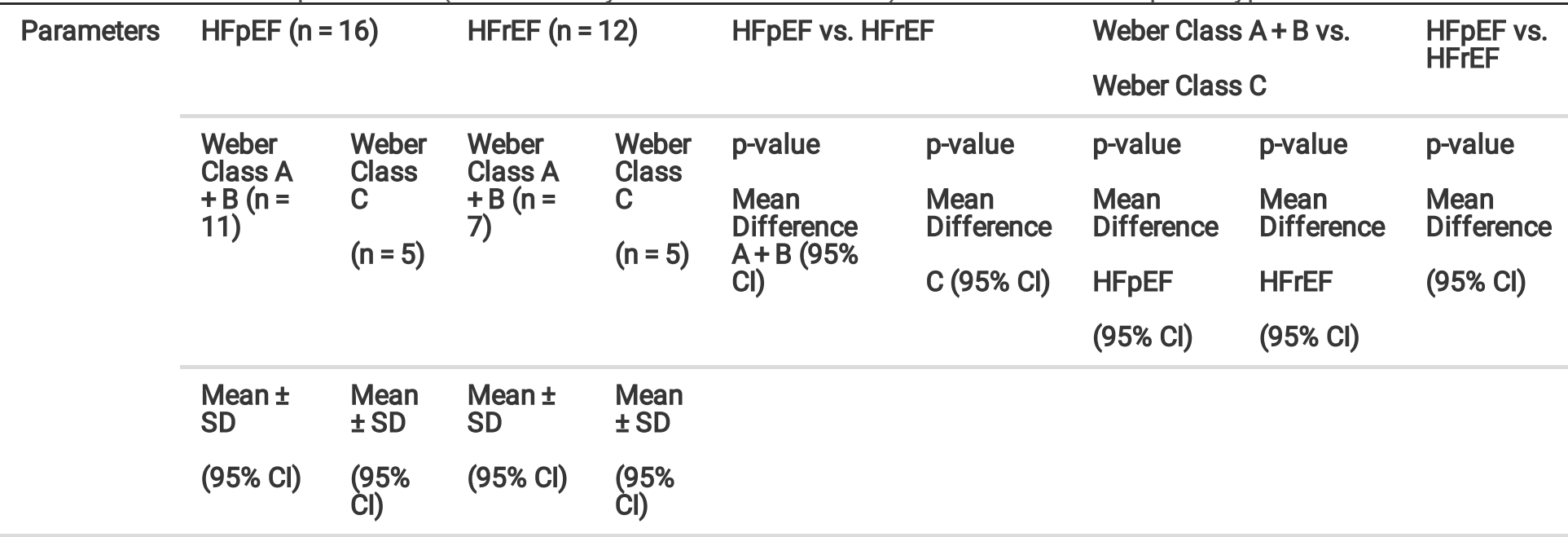

Echo

intensity, 0-

255

\begin{tabular}{|c|c|c|c|c|c|c|c|c|c|}
\hline \multirow[t]{2}{*}{$\begin{array}{l}m \text {. rectus } \\
\text { femoris }\end{array}$} & $\begin{array}{l}14.1 \pm \\
8.7\end{array}$ & $\begin{array}{l}29.7 \\
\pm 8.4\end{array}$ & $\begin{array}{l}13.1 \pm \\
5.5\end{array}$ & $\begin{array}{l}15.1 \\
\pm 6.8\end{array}$ & $0.786^{a}$ & $0.017^{a *}$ & $0.009^{a *}$ & $0.606^{a}$ & $0.140^{\mathrm{a}}$ \\
\hline & $\begin{array}{l}(8.2 \text { to } \\
19.9)\end{array}$ & $\begin{array}{l}(19.3 \\
\text { to } \\
40.2)\end{array}$ & $\begin{array}{l}(8.0 \text { to } \\
18.2)\end{array}$ & $\begin{array}{l}(6.8 \\
\text { to } \\
23.5)\end{array}$ & $\begin{array}{l}0.9(-6.2 \text { to } \\
8.1)\end{array}$ & $\begin{array}{l}14.6(3.4 \\
\text { to } 25.9)\end{array}$ & $\begin{array}{l}-15.7 \\
(-26.2 \text { to } \\
-5.2)\end{array}$ & $\begin{array}{l}-2.0(-10.6 \\
\text { to } 6.6)\end{array}$ & $\begin{array}{l}5.0(-1.8 \\
\text { to } 11.8)\end{array}$ \\
\hline
\end{tabular}

\section{Muscle \\ thickness, \\ $\mathrm{cm}$}

\begin{tabular}{|c|c|c|c|c|c|c|c|c|c|}
\hline \multirow[t]{3}{*}{$\begin{array}{l}\text { m. rectus } \\
\text { femoris }\end{array}$} & $2.1 \pm 0.5$ & $\begin{array}{l}1.7 \pm \\
0.3\end{array}$ & $1.9 \pm 0.4$ & $\begin{array}{l}1.5 \pm \\
0.5\end{array}$ & $0.435^{b}$ & $0.397^{a}$ & $0.134^{\mathrm{a}}$ & $0.145^{b}$ & $0.196^{\mathrm{a}}$ \\
\hline & $\begin{array}{l}(1.7 \text { to } \\
2.4)\end{array}$ & $\begin{array}{l}(1.3 \\
\text { to } \\
2.1)\end{array}$ & $\begin{array}{l}(1.5 \text { to } \\
2.3)\end{array}$ & $\begin{array}{l}(0.9 \\
\text { to } \\
2.1)\end{array}$ & $\begin{array}{l}0.2(-0.3 \text { to } \\
0.9)\end{array}$ & $\begin{array}{l}0.2(-0.4 \\
\text { to } 0.8)\end{array}$ & $\begin{array}{l}0.4(-0.1 \\
\text { to } 0.8)\end{array}$ & $\begin{array}{l}0.3(-0.2 \\
\text { to } 1.0)\end{array}$ & $\begin{array}{l}0.2(-0.1 \\
\text { to } 0.6)\end{array}$ \\
\hline & $(n=10)$ & $(n=4)$ & $(n=7)$ & $(n=5)$ & & & & & \\
\hline \multirow{2}{*}{$\begin{array}{l}\text { m. } \\
\text { quadriceps } \\
\text { femoris }\end{array}$} & $3.8 \pm 0.7$ & $\begin{array}{l}3.0 \pm \\
0.4\end{array}$ & \multirow{2}{*}{$\begin{array}{l}3.6 \pm 0.8 \\
(2.8 \text { to } \\
4.3)\end{array}$} & $\begin{array}{l}2.8 \pm \\
1.0\end{array}$ & $0.570^{a}$ & $0.734^{a}$ & $0.023^{a *}$ & $0.203^{a}$ & $0.364^{\mathrm{a}}$ \\
\hline & $\begin{array}{l}(3.3 \text { to } \\
4.3)\end{array}$ & $\begin{array}{l}(2.3 \\
\text { to } \\
3.6)\end{array}$ & & $\begin{array}{l}(1.6 \\
\text { to } \\
4.0)\end{array}$ & $\begin{array}{l}0.2(-0.6 \text { to } \\
1.1)\end{array}$ & $\begin{array}{l}0.2(-1.1 \\
\text { to } 1.4)\end{array}$ & $\begin{array}{l}0.8(0.1 \text { to } \\
1.5)\end{array}$ & $\begin{array}{l}0.8(-1.5 \\
\text { to } 2.0)\end{array}$ & $\begin{array}{l}0.4(-0.6 \\
\text { to } 1.3)\end{array}$ \\
\hline
\end{tabular}

Legend: Values are expressed as mean \pm standard deviation (SD), mean difference (MD), and 95\% confidence interval (95\% Cl) or median difference, number of included patients (n). Statistics: ${ }^{a}$ Unpaired t-test (MD and $95 \% \mathrm{Cl}$ ); ${ }^{\mathrm{b}}$ Mann-Whitney test (Hodge-Lehmann's median difference considered); ${ }^{*} p \leq 0.05$. Abbreviations: HFpEF, heart failure with preserved ejection fraction; HFrEF, heart failure with reduced ejection fraction; echo intensity, 0 = black, and 255 = white; m, muscle; $\mathrm{cm}$, centimeters. 
Table 5

Associations between exercise oxygenation response ( $\mathrm{TSI}, \mathrm{O}_{2} \mathrm{Hb}$ e $\left.\mathrm{HHb}, \mu \mathrm{M}\right)$ via near-infrared spectroscopy (NIRS) and echo intensity (RF_El) with isokinetic muscle strength parameter (PT, Nm), cardiorespiratory fitness (peak $\mathrm{VO}_{2}, \mathrm{~mL} \mathrm{Kg}^{-1} \cdot \mathrm{min}^{-1}$ ) and

peak power output in both HF phenotypes (HFpEF and HFrEF) according to disease severity (Weber A + B and Weber C).

\begin{tabular}{|c|c|c|c|c|c|c|c|c|c|c|}
\hline Groups & & $\begin{array}{l}\text { TSI } x \\
\text { PT }\end{array}$ & $\begin{array}{l}\mathrm{O}_{2} \mathrm{Hb} x \\
\mathrm{PT}\end{array}$ & $\mathrm{HHb} \times \mathrm{PT}$ & $\begin{array}{l}\text { TSI } x \\
\text { peak } \\
\mathrm{VO}_{2}\end{array}$ & $\begin{array}{l}\mathrm{O}_{2} \mathrm{Hb} \times \\
\text { peak } \\
\mathrm{VO}_{2}\end{array}$ & $\begin{array}{l}\mathrm{HHb} x \\
\text { peak VO }\end{array}$ & $\begin{array}{l}\text { TSIx } \\
\text { peak } \\
\text { power } \\
\text { output }\end{array}$ & $\begin{array}{l}\mathrm{O}_{2} \mathrm{Hb} x \\
\text { peak } \\
\text { power } \\
\text { output }\end{array}$ & $\begin{array}{l}\text { HHb x } \\
\text { peak } \\
\text { power } \\
\text { output }\end{array}$ \\
\hline \multirow{2}{*}{$\begin{array}{l}\text { HFpEF } \\
\text { (Weber } \\
\text { A + B) }\end{array}$} & $r$ & $-0.429^{b}$ & $-0.429^{b}$ & $0.600^{\mathrm{b}}$ & $0.118^{a}$ & $0.084^{a}$ & $0.152^{\mathrm{a}}$ & $-0.366^{a}$ & $-0.074^{a}$ & $0.378^{a}$ \\
\hline & $\mathrm{p}$ & 0.419 & 0.419 & 0.242 & 0.824 & 0.874 & 0.773 & 0.476 & 0.889 & 0.461 \\
\hline \multirow{2}{*}{$\begin{array}{l}\text { HFrEF } \\
\text { (Weber } \\
A+B)\end{array}$} & $r$ & $0.601^{a}$ & $-0.042^{a}$ & $0.009^{a}$ & $0.619^{a}$ & $-0.955^{a}$ & $-0.969^{a}$ & $0.830^{a}$ & $-0.369^{a}$ & $-0.312^{a}$ \\
\hline & $\mathrm{p}$ & 0.590 & 0.973 & 0.994 & 0.575 & 0.191 & 0.158 & 0.376 & 0.760 & 0.793 \\
\hline \multirow{2}{*}{$\begin{array}{l}\text { HFpEF } \\
\text { (Weber } \\
\text { C) }\end{array}$} & $r$ & $-0.414^{a}$ & $-0.419^{a}$ & $0.170^{\mathrm{a}}$ & $-0.621^{a}$ & $-0.915^{a}$ & $0.321^{a}$ & $0.527^{a}$ & $-0.815^{a}$ & $0.127^{a}$ \\
\hline & $\mathrm{p}$ & 0.586 & 0.581 & 0.823 & 0.380 & 0.085 & 0.679 & 0.474 & 0.185 & 0.873 \\
\hline \multirow{3}{*}{$\begin{array}{l}\text { HFrEF } \\
\text { (Weber } \\
\text { C) }\end{array}$} & $r$ & $0.339^{a}$ & $0.800^{\mathrm{b}}$ & $0.244^{\mathrm{a}}$ & $-0.196^{a}$ & $0.600^{\mathrm{b}}$ & $0.726^{a}$ & $0.616^{a}$ & $0.800^{\mathrm{b}}$ & $-0.078^{a}$ \\
\hline & $\mathrm{p}$ & 0.661 & 0.333 & 0.756 & 0.804 & 0.417 & 0.274 & 0.384 & 0.333 & 0.922 \\
\hline & & $\begin{array}{l}\text { RF_El x } \\
\text { PT }\end{array}$ & $\begin{array}{l}\text { RF_El x } \\
\text { peak } \\
\mathrm{VO}_{2}\end{array}$ & $\begin{array}{l}\text { RF_El x } \\
\text { peak } \\
\text { power } \\
\text { output }\end{array}$ & $\begin{array}{l}\text { MT_RF } \\
\text { x PT }\end{array}$ & $\begin{array}{l}\text { MT_RF } \\
\text { xpeak } \\
\mathrm{VO}_{2}\end{array}$ & $\begin{array}{l}\text { MT_RF x } \\
\text { peak } \\
\text { power } \\
\text { output }\end{array}$ & & & \\
\hline \multirow{2}{*}{$\begin{array}{l}\text { HFpEF } \\
\text { (Weber } \\
A+B)\end{array}$} & $r$ & $-0.331^{b}$ & $-0.068^{a}$ & $0.031^{a}$ & $0.082^{b}$ & $0.599^{a}$ & $0.575^{a}$ & & & \\
\hline & $\mathrm{p}$ & 0.320 & 0.842 & 0.929 & 0.811 & 0.052 & 0.068 & & & \\
\hline \multirow{2}{*}{$\begin{array}{l}\text { HFrEF } \\
\text { (Weber } \\
A+B)\end{array}$} & $r$ & $-0.036^{b}$ & $0.018^{b}$ & $0.179^{b}$ & $0.593^{b}$ & $0.187^{b}$ & $0.297^{b}$ & & & \\
\hline & $\mathrm{p}$ & 0.964 & 0.988 & 0.713 & 0.174 & 0.701 & 0.529 & & & \\
\hline \multirow{2}{*}{$\begin{array}{l}\text { HFpEF } \\
\text { (Weber } \\
\text { C) }\end{array}$} & $r$ & $0.121^{a}$ & $-0.910^{a}$ & $-0.081^{a}$ & $0.405^{\mathrm{a}}$ & $0.468^{a}$ & $0.108^{a}$ & & & \\
\hline & $\mathrm{p}$ & 0.847 & $0.032^{a *}$ & 0.897 & 0.499 & 0.426 & 0.093 & & & \\
\hline \multirow{2}{*}{$\begin{array}{l}\text { HFrEF } \\
\text { (Weber } \\
\text { C) }\end{array}$} & $r$ & $-0.228^{a}$ & $-0.430^{a}$ & $-0.125^{a}$ & $0.880^{a}$ & $0.613^{a}$ & $0.900^{\mathrm{a}}$ & & & \\
\hline & $\mathrm{p}$ & 0.713 & 0.470 & 0.842 & $0.049 *$ & 0.271 & 0.083 & & & \\
\hline \multicolumn{11}{|c|}{$\begin{array}{l}\text { Legend: Values are expressed as absolute values. Statistics: }{ }^{\text {a }} \text { Person's correlation test ( } \mathrm{r} \text { correlation coefficient with } 0.00 \text { no } \\
\text { association; } 0.20 \text { weakly; } 0.50 \text { moderately; } 0.8 \text { strongly and } 1.00 \text { perfectly) and }{ }^{b} \text { Spearman's correlation test (rho correlation } \\
\text { coefficient with } 0.00 \text { to } 0.20 \text { negligible; } 021 \text { to } 0.40 \text { weak; } 0.41 \text { to } 0.60 \text { moderate; } 0.61 \text { to } 0.80 \text { strong and } 0.81 \text { to } 1.00 \text { very } \\
\text { strong); }{ }^{*} \mathrm{p} \leq 0.05 \text {, correlation coefficient of } 0.40-0.59 \text { were considered moderate. Abbreviations: TSI, tissue saturation index; } \\
\text { PT, peak torque; } \mathrm{VO}_{2} \text {, oxygen uptake; } \mathrm{O}_{2} \mathrm{Hb} \text {, oxyhemoglobin; } \mathrm{HHb} \text {, deoxyhemoglobin; HFpEF, heart failure with preserved } \\
\text { ejection fraction; } \mathrm{HFrEF} \text {, heart failure with reduced ejection fraction; RF, rectus femoris muscle; El, echo intensity. }\end{array}$} \\
\hline
\end{tabular}

\section{Discussion}

Our study found poor peripheral muscle microcirculation dynamics, particularly in HFpEF patients during isokinetic muscle strength testing, which was more pronounced in Weber $\mathrm{C}$ patients. Also, HFrEF with Weber Class $\mathrm{A}+\mathrm{B}$ presented a worse recovery than HFpEF following the rationale that HFrEF presents better $\mathrm{O}_{2}$ extraction during strength exercise than HFpEF, which seems to justify why HFrEF recovery worsens than HFpEF for the HHb parameter. Moreover, although lower limb muscle strength and ultrasound-derived thickness were not different between subgroups, echo intensity revealed worse muscle quality than HFpEF patients. This was negatively associated with cardiorespiratory fitness in the same phenotype. Hence, our preliminary findings 
suggest that peripheral muscle microcirculation dynamics can affect a strength-type exercise similar to an aerobic-type exercise in HFpEF.

A similar local oxygen response decrease in HFpEF was also observed during a cardiopulmonary exercise test (CPET), highlighting the significant role of impaired arteriovenous $\mathrm{O}_{2}$ difference augmentation in contributing to exercise intolerance in the HFpEF population [19]. Moreover, the arteriovenous $\mathrm{O}_{2}$ difference is also reduced in HFpEF when performing a hand dynamometer test [46]. Another study evaluated the oxygen response during plantar flexion exercise by magnetic resonance with spectroscopy in HFpEF patients and healthy individuals, reveling a poor performance in HFpEF patients, indicated by a faster decrease in phosphocreatine and consequent impairment in the ATP flow [47,48], possible causing microvascular damage [49]. Our findings demonstrated that Weber Class $C$ patients presented a reduced capability to reach a greater oxygen extraction during exercise in HFpEF compared to Classes A and B during an isokinetic muscle strength test. Moreover, an extended recovery duration was found in HFpEF in both severities classes. A previous study comparing HFpEF and healthy controls identified that the major mechanism underlying the functional impairment in such group appears to be related to deranged peripheral hemodynamics, including a reduced leg blood flow and vascular conductance [50]. The current study thus adds to previous research that HFpEF patients experience significant abnormalities in skeletal muscle oxygen extraction during strength-type exercises. The latter could contribute to exercise intolerance, particularly when greater (skeletal muscle) exercise intensities are elicited.

Higher echo intensity values have been related to an increased intramuscular adipose and connective tissue distribution [51-53]. Previous studies have shown that HFpEF presents $30 \%$ higher fat tissue and intramuscular fat tissue than the healthy population $[54,23,55]$. Our study arouses new findings regarding muscular quality differences between HFpEF and HFrEF. Considering all HF severities, our study has found an increasing trend of the RF echo intensity in the HFpEF phenotype with Weber Class C. Interestingly; the higher echo intensity was associated with a lower peak $\mathrm{VO}_{2}$ in $\mathrm{HFpEF}$ participants in the Weber $\mathrm{C}$ severity subgroup. This could demonstrate different mechanisms related to exercise intolerance in HFpEF, suggesting a closer association between muscle quality, disease severity, and exercise intolerance. Furthermore, Nakano et al. [56] described a positive correlation between quadriceps femoris echo intensity and NYHA class and age. A negative correlation between quadriceps femoris echo intensity and peak $\mathrm{VO}_{2}$ was also described in $\mathrm{HF}$ and healthy subjects [56].

A greater quantity of type II muscle fiber in $\mathrm{HF}$ and the lower capillarity ratio per fiber were already related to greater exercise intolerance in previous studies [14,23]. Similarly, a lower amount of type I fiber has been shown to cause a lower peak $\mathrm{VO}_{2}[14,23]$. Furthermore, the reduced oxidative and diffusive capacity combined with a low exercise tolerance on HF patients compared to healthy volunteers suggests that skeletal muscle metabolism is a potentially important target for future HF treatment strategies $[48,57,58]$, providing more assertive and individualized treatment strategies.

This study contains limitations that might be addressed. First, a limited study population may reduce the results' external validity, and the results should be interpreted with caution. However, this study presents for the first time microvascular dynamics during strength exercise, helping to understand exercise intolerance in heart failure. Also, despite considering clinical signs, symptoms, and echocardiographic data to justify the clinical diagnosis, not all patients had their BNP tested; however, the patients were evaluated and diagnosed by cardiologists. Finally, considering this is the first study evaluating local oxygen extraction during isokinetic muscle strength and echo intensity in HFrEF vs. HFpEF, these findings add new insights future well-designed randomized controlled trials are needed to understand better the effects of the peripheral muscle microcirculation dynamics during strength exercise testing in heart failure patients.

\section{Conclusions}

Despite similar isokinetic muscle strength between HFpEF and HFrEF, our study reveals a pronounced microcirculatory impairment and slower recovery following a during isokinetic muscle strength testing in $\mathrm{HFpEF}$, coupled with ultrasound-detectable musculoskeletal abnormalities, which was also strongly associated with cardiorespiratory capacity, particularly in those more severely affected (Weber $\mathrm{C}$ ). Oxidative and structural muscle impairment is a possible underlying exercise intolerance mechanism, which appears able to impact strength modality in HFpEF.

\section{Declarations}

Page $15 / 22$ 


\section{Acknowledgments}

The authors acknowledge all patients' availability and the research group (GPRC) that made this study possible. We also acknowledge the laboratory coordinators from the University of Brasília (Laboratório de Fisiologia e Laboratório de Treinamento de Força da Universidade de Brasília), MEDCOR clinic for the echocardiographic images assessments, and Ms. Robson Borges who have helped to recruit patients.

\section{Author information}

\section{Affiliations}

\section{University of Brasilia, Brasilia, DF, Brazil}

Amanda Vale-Lira, Natália Turri-Silva, João Luiz Quagliotti Durigan \& Gerson Cipriano Júnior

Universidade Evangélica de Goiás, Anápolis, GO, Brazil

Gaspar R. Chiappa

\section{Hasselt University, Faculty of Rehabilitation Sciences - BIOMED-REVAL (Rehabilitation Research Centre), Diepenbeek, Belgium}

Natália Turri-Silva, Kenneth Verboven \& Dominique Hansen

\section{Heart Centre Hasselt, Jessa Hospital, Hasselt, Diepenbeek, Belgium}

Dominique Hansen

Author contributions

AVL, NTS, and GCJ conceived the study design; AVL and NTS conducted the study; AVL analyzed the results; AVL, NTS, KV, JQD, GRC, DH, and GCJ, interpreted of data. All authors reviewed the manuscript and approved the submitted version.

\section{Competing interests}

The authors declare no competing interests.

\section{Data availability}

The datasets generated during or analyzed during the current study are available from the corresponding author on reasonable request.

\section{References}

1. 1. Naghavi, M. et al. Global, regional, and national age-sex specific all-cause and cause-specific mortality for 240 causes of death, 1990-2013: a systematic analysis for the Global Burden of Disease study 2013. Lancet. 385, 117 - 71; 10.1016/S0140-6736(14)61682-2

2. 2. World Health Organization. Cardiovascular diseases: key facts https://www.who.int/news-room/factsheets/detail/cardiovascular-diseases-(cvds) (2017).

3. 3. Junior, G.C., Neves, L. M. T., Cipriano, G. F. B., Chiappa, G. R. \& Borghi-Silva, A. Cardiovascular disease prevention and implications for worksite health promotion programs in Brazil. Prog Cardiov Dis. 56, 943 - 500 (2014); 10.1016/j.pcad.2013.10.018

4. 4. Heidenreich, P. A. et al. Forecasting the impact of heart failure in the United States. Circ Heart Fail. 6, 606-619 (2013); 10.1161/HHF.0b013e318291329

5. 5. Ponikowski, P., et al. 2016 ESC Guidelines for the diagnosis and treatment of acute and chronic heart failure: the task force for the diagnosis and treatment of acute and chronic heart failure of the European Society of Cardiology (ESC). Eur J Heart 
Fail. 18, 891-975 (2016); 10.1093/eurheartj/ehw128

6. 6. Redfield, M. M. Heart failure with preserved ejection fraction. N Engl J Med. 375, 1868-1877 (2016); 10.1056/NEJMcp1511175

7. 7. Dhingra, A. et al. Epidemiology of heart failure with preserved ejection fraction. Curr Heart Fail Rep. 11, 354-365 (2014); 10.1007/s11897-014-0223-7

8. 8. Owan T.E., et al. Trends in prevalence and outcome of heart failure with preserved ejection fraction. $N$ Engl J Med. 355, 251-259 (2006); 10.1056/NEJMoa052256

9. 9. Kemp, C. D. \& Conte, J. V. The pathophysiology of heart failure. Cardiovasc Pathol. 21, 365-371 (2012); 10.1016/j.carpath.2011.11.007

10. 10. Esposito, F., Mathieu-Costello, O., Shabetai, R., Wagner, P. D. \& Richardson, R. S. Limited maximal exercise capacity in patients with chronic heart failure: partitioning the contributors. J Am Coll Cardiol. 55, 1945-1954 (2010); 10.1016/j.jacc.2009.11.086

11. 11. Bocchi, E. A., et al. Sociedade Brasileira de Cardiologia. Atualização da Diretriz Brasileira de Insuficiência Cardíaca Crônica - 2012. Arq Bras Cardiol. 98, 1-33 (2012); pdf/abc/v93n1s1/abc93_1s1.pdf

12. 12. Poole, D.C., Richardson, R.S., Haykowsky, M.J., Hirai, D.M. \& Musch, T.I. Exercise limitations in heart failure with reduced and preserved ejection fraction. J Appl Physiol. 1, 208-224 (2018); 10.1152/japplphysiol.00747.2017

13. 13. Coats, A. J. S., Clark, A. L., Piepoli, M., Volterrani, M. \& Poole-Wilson, P. A. Symptoms and quality of life in heart failure: the muscle hypothesis. Br Heart J. 72, 36-39 (1994); 10.1136/hrt.72.2_Suppl.S36

14. 14. Adams, V., Linke, A. \& Winzer, E. Skeletal muscle alterations in HFrEF vs. HFpEF. Curr Heart Fail Rep. 14, 489-497 (2017); $10.1007 / \mathrm{s} 11897-017-0361-9$

15. 15. Kan Van, G. A. Epidemiology and consenquences of sarcopenia. J Nutr Health Aging. 13, 708-712 (2009); $10.1007 /$ s12603-009-0201-z

16. 16. Bekfani, T. et al. Sarcopenia in patients with heart failure with preserved ejection fraction: Impact on muscle strength, exercise capacity and quality of life. Inter J Cardiol. 222, 41-46 (2016); 10.1016/j.ijcard.2016.07.135

17. 17. Poole, D. C., Hirai, D. M., Copp, S. W. \& Musch, T. I. Muscle oxygen transport and utilization in heart failure: implications for exercise (in)tolerance. Am J Physiol Heart Circ Physiol. 302, H1050-1063 (2012); 10.1152/ajpheart.00943.2011

18. 18. Haykowsky, M. J., et al. Determinants of exercise intolerance in elderly heart failure patients with preserved ejection fraction. J Am Coll Cardiol. 58, 265-274 (2011); 10.1016/j.jacc.2011.02.055

19. 19. Dhakal, B. P., et al. Mechanisms of exercise intolerance in heart failure with preserved ejection fraction: the role of abnormal peripheral oxygen extraction. Circ Heart Fail. 8, 286-294 (2015); 10.1161/CIRCHEARTFAILURE.114.001825

20. 20. Wilson, J.R., Mancini, D.M. \& Dunkman, W.B. Exertional fatigue due to skeletal muscle dysfunction in patients with heart failure. Circulation. 87, 470-475 (1993); 10.1161/01.CIR.87.2.470

21. 21. Borlaug, B. A., et al. Global cardiovascular reserve dysfunction in heart failure with preserved ejection fraction. J Am Coll Cardiol. 56, 845-854 (2010); 10.1016/j.jacc.2010.03.077

22. 22. Boyes, N. G., et al. Effects of heavy-intensity priming exercise on pulmonary oxygen uptake kinetics and muscle oxygenation in heart failure with preserved ejection fraction. Am J Physiol Regul Integr Comp Physiol. 316, 199-209 (2019); https://doi.org/10.1152/ajpregu.00290.2018

23. 23. Kitzman, D.W., et al. Skeletal muscle abnormalities and exercise intolerance in older patients with heart failure and preserved ejection fraction. Am J Physiol Heart Circ Physiol. 306, H1364-1370 (2014); 10.1152/ajpheart.00004.2014

24. 24. Haykowsky, M. J., et al. Regional adipose distribution and its relationship to exercise intolerance in older obese patients who have heart failure with preserved ejection fraction. JACC Heart Fail. 6, 640-649 (2018);

https://doi.org/10.1016/j.jchf.2018.06.002

25. 25. Niemeijer, V. M. Skeletal muscle characteristics and exercise intolerance in chronic heart failure. Eindhoven: Technische Universiteit Eindhoven. https://research.tue.nl (2018).

26. 26. Marti, C. N., et al. Endothelial dysfunction, arterial stiffness and heart failure. JACC. 60, 1455-1469 (2012); https://doi.org/10.1016/j.jacc.2011.11.082

Page $17 / 22$ 
27. 27. O'Connor, C. M., et al. Factors related to morbidity and mortality in patients with chronic heart failure with systolic dysfunction the HF-ACTION predictive risk score model. Circ Heart Fail. 5, 63-71 (2012);

10.1161/CIRCHEARTFAILURE.111.963462

28. 28. Montero D., Diaz-Cañestro C. Determinants of exercise intolerance in heart failure with preserved ejection fraction: $A$ systematic review and meta-analysis. Int J Cardiol. 254, 224-229 (2018); 10.1016/j.ijcard.2017.10.114

29. 29. Malta, M., Cardoso, L.O., Bastos, F.I., Magnanini, M.M.F. \& da Silva C.M.F.P. STROBE initiative: guidelines on reporting obsevartional studies. Rev Saúde Pública. 44, 559-565 (2010). 10.1590/S0034-89102010000300021

30. 30. Yancy, C.W., et al. 2013 ACCF/AHA guideline for the management of heart failure: a report of the American College of Cardiology Foundation/American Heart Association task force on practice guidelines. J Am Coll Cardiol. 62, e147-239 (2013); https://doi.org/10.1016/j.jacc.2013.05.019

31. 31. Guazzi, M., et al. 2016 focused update: clinical recommendations for cardiopulmonary exercise testing data assessment in specific patient populations. Eur Heart J. 39, 1144-1161 (2018); 10.1093/eurheartj/ehw180.

32. 32. Toth, M.J., et al. Reduced knee extensor function in heart failure is not explained by inactivity. Int J Cardiol. 143, 276-282 (2010); https://doi.org/10.1016/j.ijcard.2009.02.040

33. 33. Toth, M.J., Miller, M.S., Ward, K.A. \& Ades, P.A. Skeletal muscle mitochondrial density, gene expression, and enzyme activities in human heart failure: minimal effects of the disease and resistance training. $J$ Appl Physiol (1985). 112, 18641874 (2012); 10.1152/japplphysiol.01591.2011

34. 34. Stevens, A.L.M., et al. Exercise training improves insulin realease during glucose tolerance testing in stable chronic heart failure patients. J Cardio Rehabil Prev. 35, 37-46 (2015); 10.1097/HCR.0000000000000092

35. 35. Delagardelle, C., Feiereisen, P., Krecké, R., Essamri, B. \& Beissel, J. Objective effects of a 6 months' endurance and strength training program in outpatients with congestive heart failure. Med Sci Sports Exerc. 31, 1102-1107 (1999);

10.1097/00005768-199908000-00004

36. 36. Delagardelle, C., et al. Strength/endurance training versus endurance training in congestive heart failure. Med Sci Sports Exerc. 34, 1868-1872 (2002); 10.1097/00005768-200212000-00002

37. 37. Minotti, J.R., et al. Impaired skeletal muscle function in patients with congestive heart failure: relationship to systemic exercise performance. J Clin Invest. 88, 2077-82 (1991); 10.1172/JCI115537

38. 38. Owen-Reece, H., Smith, M., Elwell, C.E. \& Goldstone, J.C. Near infrared spectroscopy. Br J Anaesth. 82, 418-426 (1999); https://watermark.silverchair.com/418.pdf

39. 39. Alvares T.S., Oliveira G.V; Soares R. \& Murias M. Near-infrared spectroscopy-derived total haemoglobin as an indicator of changes in muscle blood flow during exercise-induced hyperaemia. J Sports Sci. 38, 751-758 (2020);

$10.1080 / 02640414.2020 .1733774$

40. 40. Tillquist, M., et al. Bedside ultrasound is a practical and reliable measurement tool for assessing quadriceps Muscle layer thickness. J Parenter Enter Nutr. 38, 886-890 (2013); 10.1177/0148607113501327

41. 41. Abràmoff, M.D., Magalhães, P.J. \& Ram, S.J. Image processing with ImageJ. Biophotonics Int. 11, 36-42 (2005). publications/download/bio2004.pdf

42. 42. Arts, I. M. P., Phillens, S., Schelhaas, H. J., Overeem, S. \& Zwarts, M. J. Normal values for quantitative muscle ultrasonography in adults. Muscle Nerve. 41, 32-41(2010); 10.1002/mus.21458

43. 43. Blazevich, A.J., Gill, N.D. \& Zhou, S. Intra- and intermuscular variation in human quadriceps femoris architecture assessed in vivo. J Anat. 209, 289-310 (2006); 10.1111/j.1469-7580.2006.00619.x

44. 44. Zou K. H., Tuncali, K. \& Silverman, S. G. Correlation and simple linear regression. Radiology. 227, 617-622 (2003); 10.1148/radiol.2273011499

45. 45. Prion, S. \& Haerling, K. A. Making sense of methods and measurement: Spearman-rho ranked-order correlation coefficient. Clinical Simulation in Nursing. 10, 535-536 (2014); 10.1016/j.ecns.2014.07.005

46. 46. Zamani P., et al. Peripheral determinants of oxygen utilization in heart failure with preserved ejection fraction: central role of adiposity. JACC Basic Trans/ Sci. 5, 211-225 (2020); 10.1016/j.jacbts.2020.01.003

Page $18 / 22$ 
47. 47. Bhella P.S., et al. Abnormal haemodynamic response to exercise in heart failure with preserved ejection fraction. Eur $J$ Heart Fail. 13, 1296-1304 (2011); 10.1093/eurjhf/hfr133

48. 48. Weiss K., et al. Fatigability, exercise intolerance and abnormal skeletal muscle energetics in heart failure. Cir Heart Fail. 10, e004129 (2017); 10.1161/CIRCHEARTFAILURE.117.004129

49. 49. Tucker W.J., et al. Pathophysiology of exercise intolerance and its treatment with exercise-based cardiac rehabilitation in heart failure with preserved ejection fraction. J Cardiopulm Rehabil Prev. 40, 9-16(2020); 10.1097/HCR.0000000000000481

50. 50. Weavil, J.C., et al. Heart failure with preserved ejection fraction diminishes peripheral hemodynamics and accelerates exercise-induced neuromuscular fatigue. Am J Physiol Heart Circ Physiol. 320, H338-H351 (2021);

10.1152/ajpheart.00266.2020

51. 51. Young, H., Jenkins, N.T., Zhao, Q. \& Mccully, KK. Measurement of intramuscular fat by muscle echo intensity. Muscle Nerve. 6, 963-971 (2015); 10.1002/mus.24656

52. 52. Pillen, S., et al. Skeletal muscle ultrasound: correlation between fibrous tissue and echo intensity. Ultrasound in Med. \& Biol. 35, 443-446 (2009); 10.1016/j.ultrasmedbio.2008.09.016

53. 53. Schaufelberger, M., Eriksson, B.O., Grimby, G., Held, P. \& Swedberg, K. Skeletal muscle fiber composition and capillarization in patients with chronic heart failure: relation to exercise capacity and central hemodynamics. J Card Fail. 1, 267-272 (1995); 10.1016/1071-9164(95)90001-2

54. 54. Haykowsky, M.J., et al. Impaired aerobic capacity and physical functional performance in older heart failure patients with preserved ejection fraction: role of lean body mass. J Gerontol A Biol Sci Med Sci. 68, 968-975 (2013);

10.1093/gerona/glt011

55. 55. Haykowsky, M.J., et al. Skeletal muscle composition and its relation to exercise intolerance in older patients with heart failure and pre- served ejection fraction. Am J Cardiol. 113, 1211-1216 (2014); 10.1016/j.amjcard.2013.12.031

56. 56. Nakano, l., et al. Enhanced echo intensity of skeletal muscle is associated with exercise intolerance in patients with heart failure. J Card Fail. 8, 685-693 (2020); 10.1016/j.cardfail.2019.09.001

57. 57. Molina, A.J.A., et al. Skeletal muscle mitochondrial content, oxidative capacity, and Mfn2 expression are reduced in older patients with heart failure and preserved ejection fraction and are related to exercise intolerance. J Am Coll Cardiol. 8, $636-45$ (2016); 10.1016/j.jchf.2016.03.011

58. 58. Del Buono, M.G., et al. Exercise intolerance in patients with heart failure: JACC State-of-the-art-review. J Am Coll Cardiol. 73, 2209-2225 (2019); 10.1016/j.jacc.2019.01.072

\section{Figures}


Weber A + B

HFpEF

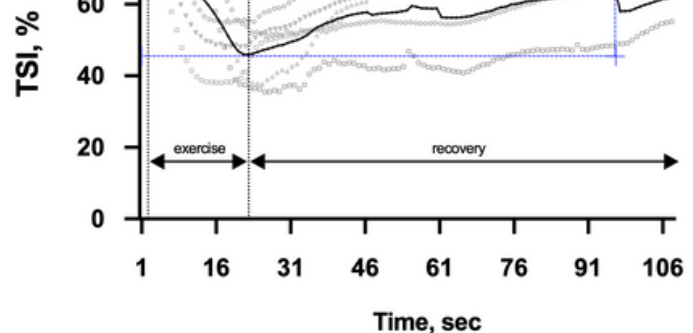

HFrEF

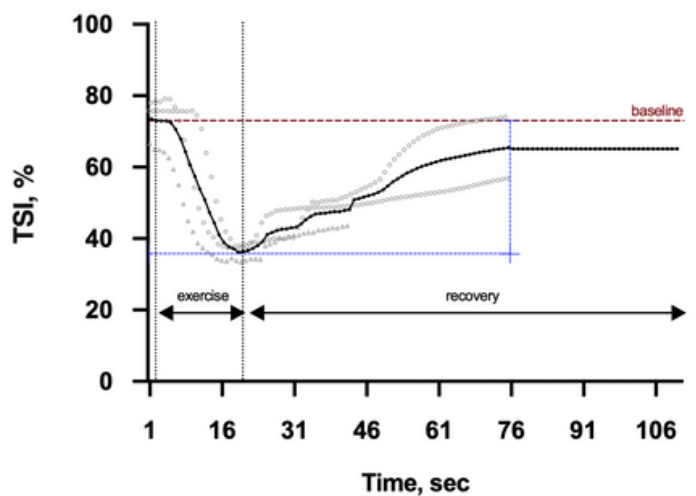

Weber C
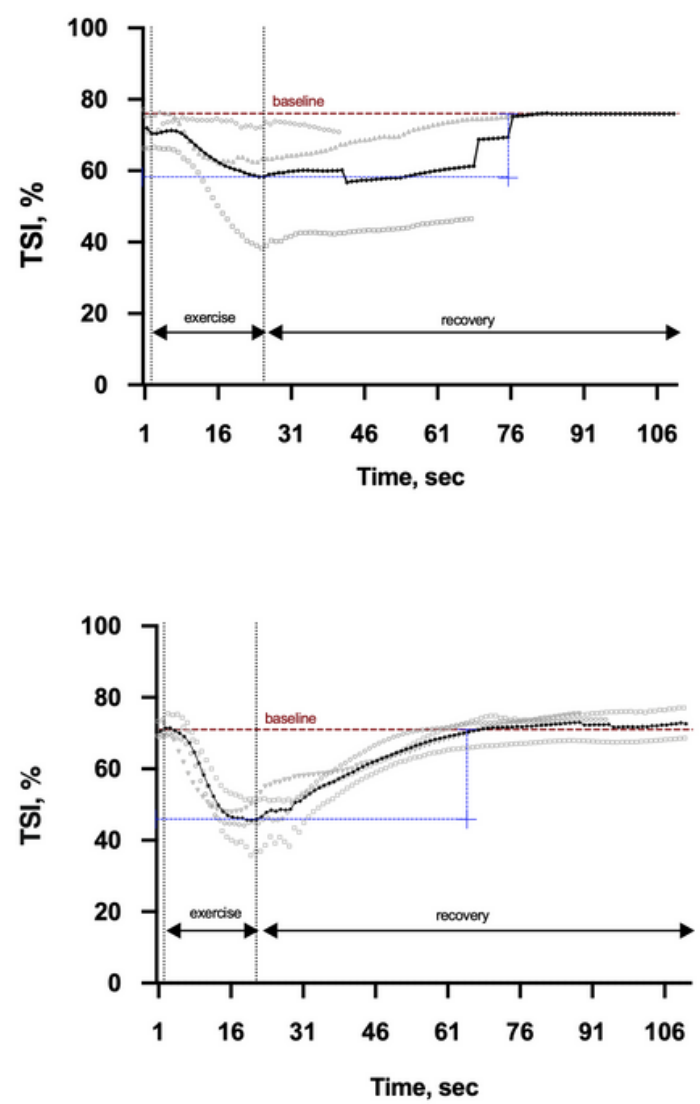

\section{Figure 1}

Representative cases of local oxygen extraction (tissue saturation index - TSI, \%) during isokinetic muscle strength evaluation by the Weber Class in both heart failure phenotypes. Average and individual behavior of local oxygen extraction (tissue saturation index) during the isokinetic muscle strength maneuver by the Weber Class A+B or C between heart failure patients' groups. HFpEF, heart failure with preserved ejection fraction; HFrEF, heart failure with reduced ejection fraction; TSI, tissue saturation index; sec, seconds. 
Weber A + B

HFpEF
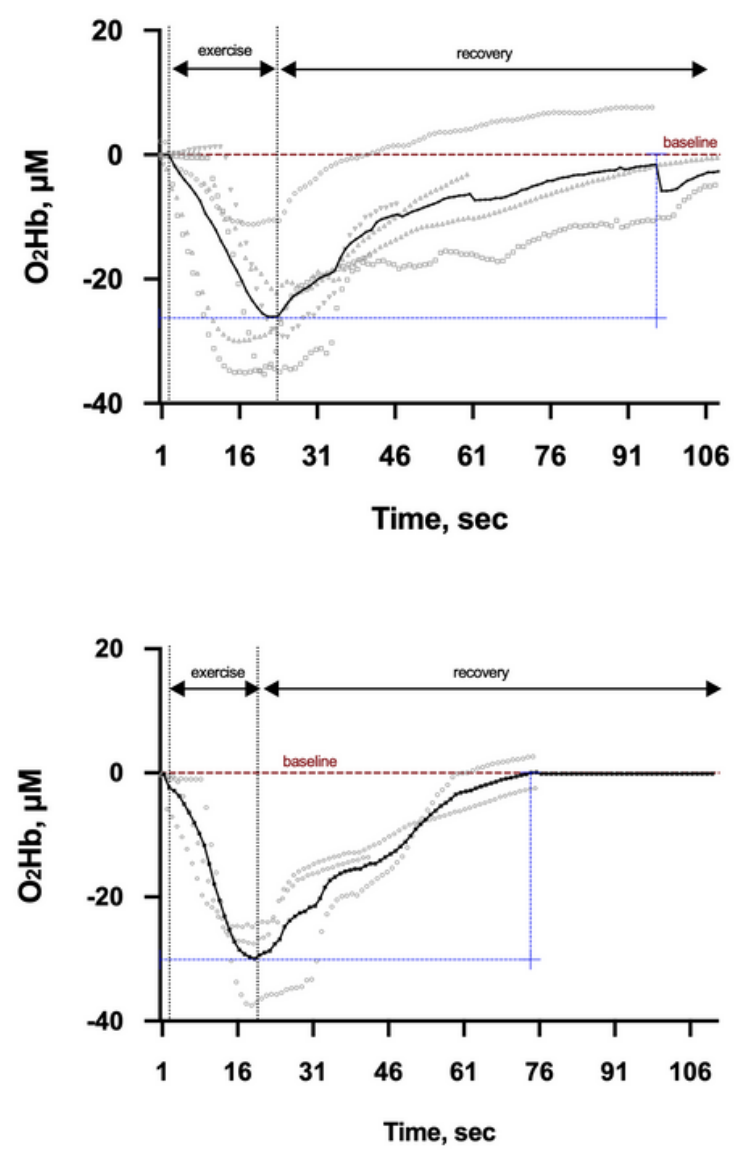

Weber C
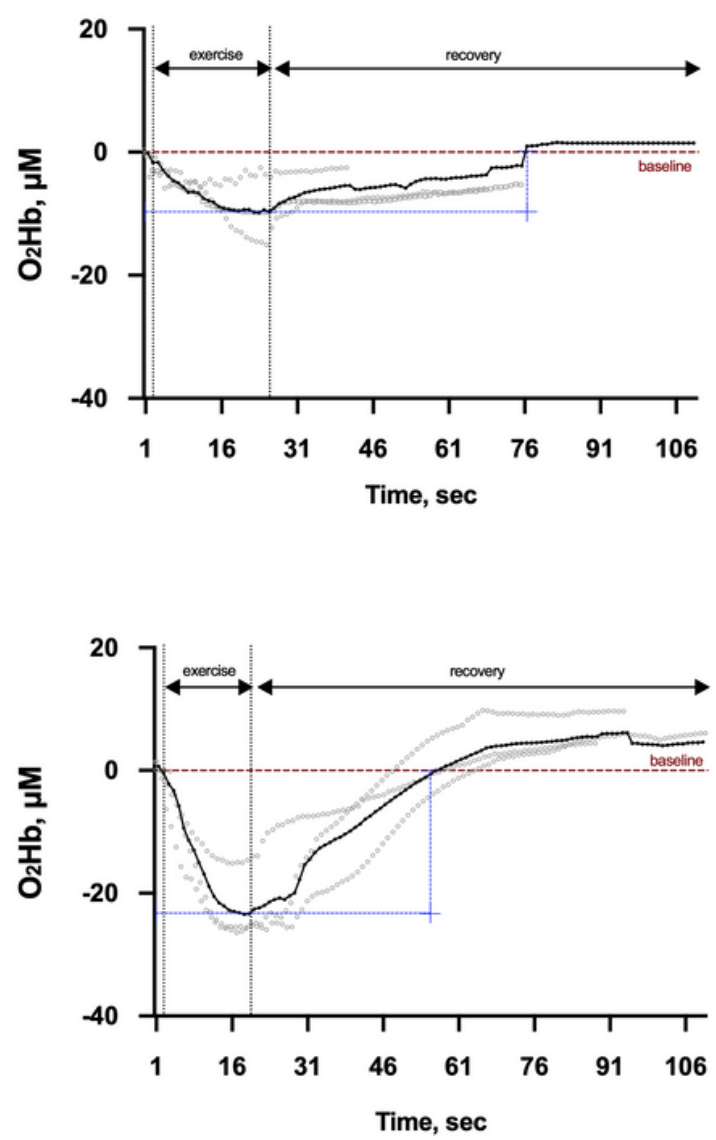

Figure 2

Representative cases of local oxygen extraction (oxygenation - $02 \mathrm{Hb}, \mu \mathrm{M}$ ) during isokinetic muscle strength evaluation by the Weber Class in both heart failure phenotypes. Average and individual behavior of local oxygen extraction (oxygenation) during the isokinetic muscle strength maneuver by the Weber Class A+B or C between heart failure patients' groups. HFpEF, heart failure with preserved ejection fraction; $\mathrm{HFrEF}$, heart failure with reduced ejection fraction; $\mathrm{O} 2 \mathrm{Hb}$, oxyhemoglobin; sec, seconds; $\mu \mathrm{M}$, micrometer. 
Weber A + B
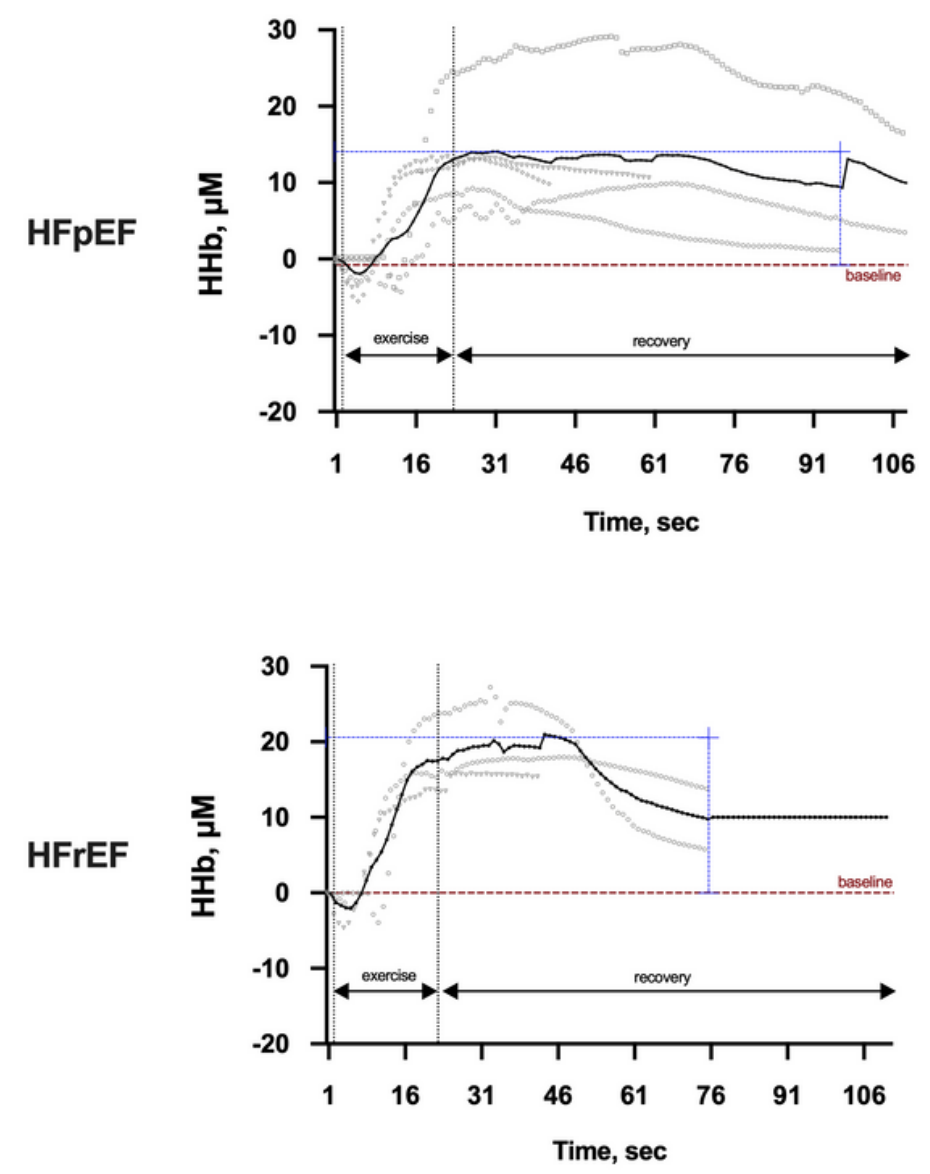

Weber C
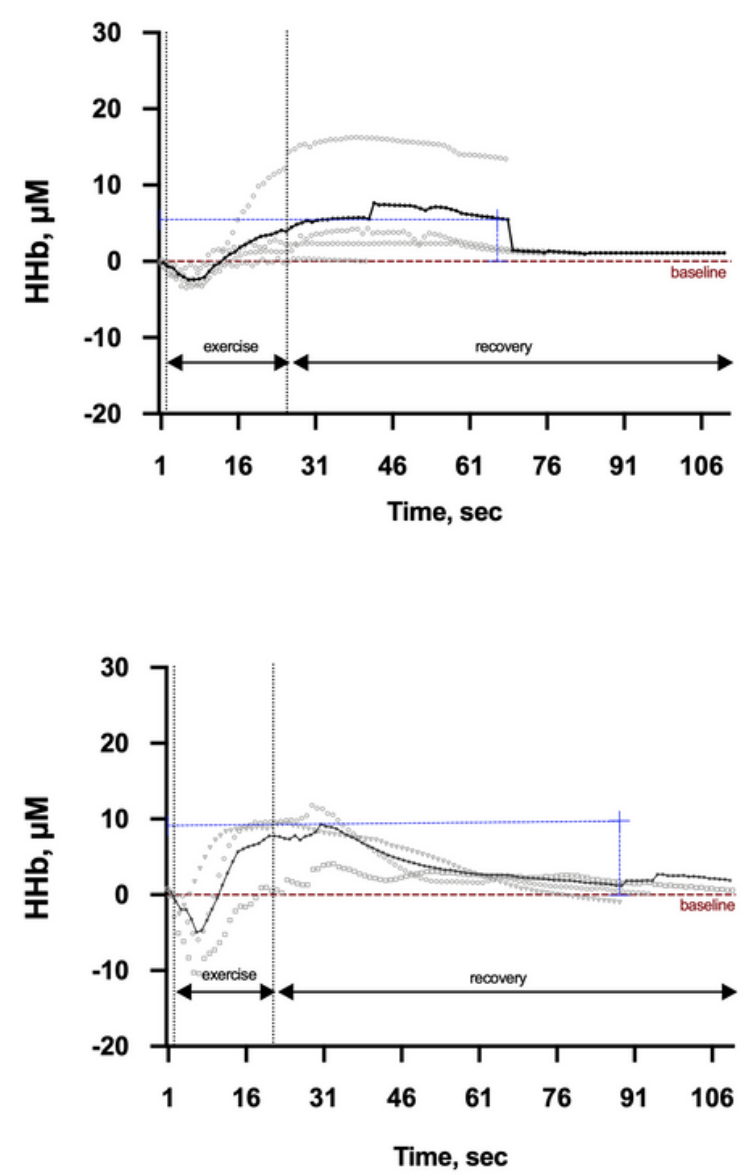

\section{Figure 3}

Representative cases of local oxygen extraction (deoxygenation - $\mathrm{HHb}, \mu \mathrm{M}$ ) during isokinetic muscle strength evaluation by the Weber Class in both heart failure phenotypes. Average and individual behavior of local oxygen extraction (deoxygenation) during the isokinetic muscle strength maneuver by the Weber Class A+B or C between heart failure patients' groups. HFpEF, heart failure with preserved ejection fraction; $\mathrm{HFrEF}$, heart failure with reduced ejection fraction; $\mathrm{HHb}$, deoxyhemoglobin; sec, seconds; $\mu \mathrm{M}$, micrometer.

\section{Supplementary Files}

This is a list of supplementary files associated with this preprint. Click to download.

- SupplementaryMaterial.pdf 